Article

\title{
Understanding Innovation for Sustainable Business Management Capabilities and Competencies under Uncertainty
}

\section{Kuo-Jui Wu ${ }^{1}$, Ching-Jong Liao ${ }^{1}$, Ming-Lang Tseng ${ }^{2, *}$ and Pei-Jay Chou ${ }^{2}$}

1 Department of Industrial Management, National Taiwan University of Science and Technology, Taipei 10607, Taiwan; E-Mails: D10101011@mail.ntust.edu.tw (K.-J.W.); cjliao@mail.ntust.edu.tw (C.-J.L.)

2 Department of Business Administration, Lunghwa University of Science and Technology, No. 300, Sec. 1, Wanshou Road, Taoyuan County 33306, Taiwan; E-Mail: G1042231012@mail.1hu.edu.tw

* Author to whom correspondence should be addressed; E-Mail: ml.tseng@mail.lhu.edu.tw; Tel.: +886-910-309-400.

Academic Editor: Kannan Govindan

Received: 7 July 2015 / Accepted: 8 October 2015 / Published: 12 October 2015

\begin{abstract}
In recent years, many firms have come to understand that innovation is an important issue in sustainable business management, as it helps improve firm capabilities and competencies. Because of the fiercely competitive environment in the hotel industry, innovation has become a critical factor in the process of hotel differentiation, leading to sustainable business success. However, the literature has not thoroughly examined the role of innovation or the hierarchical structure of the capabilities and competencies in sustainable business management. This study adopts interval-valued triangular fuzzy numbers and grey relational analysis to provide a competitive priority ranking for the aspects and criteria that assist firms in decision-making. The study results indicate that innovation in technology capabilities and networking and social capabilities - in addition to competencies in systemic thinking - are the most important aspects of sustainable business management. In particular, this study indicates that to succeed in building a sustainable business in the hotel industry, firms should upgrade and integrate their business technologies, collaborate with actors inside and outside the firm, build trust as well as a shared vision that includes common agreement, and develop competencies in inventive thinking to support innovation and foster changes in strategy, structure, administrative procedures, and systems when necessary.
\end{abstract}


Keywords: sustainable business management; innovation; capabilities and competences; interval-valued triangular fuzzy numbers; grey relational analysis

\section{Introduction}

In the era of globalization, the hotel industry has become more cut-throat as a result of increased competition from more firms [1-3]. To compete, hotels must enhance their capabilities and competencies to improve operational performance in myriad ways as judged under various criteria. Thus, hotels must apply new technologies to track and respond to customer demands and achieve sustainability $[3,4]$. Sustainability will be achieved based on innovation in response to customer needs for new services and products, which will lead to increased market share and profits and will also contribute to business sustainability [5-7]. However, innovation involves increased risk, and success is not guaranteed; to enjoy a higher likelihood of success in innovation, thorough assessments must be undertaken and value-creating investments must be chosen [8-11].

In the literature, innovation is considered an important element of firm success [12]. Harper and Becker [13] indicated that innovation resulted in significant change-preferably an improvement in the real product, process, or service - that exceeds the impact of previous achievements; these authors further indicated that innovation supported sustainable business management. Firms encourage innovation to achieve production and marketing goals, to improve product or service quality, to lower their operational costs, to increase their market share, to attain production flexibility, and to improve the management process [14]. Previous studies have generally indicated that innovation was positively associated with business sustainability [15,16]. However, Zahra [17] and Colquitt et al. [18] also noted that innovation may be a risky investment; developing and launching new products and/or services is necessary for firm survival and sustainability, but these are costly business processes. Delgado [19] argued that the positive effects of innovation, particularly technology innovation, may be exaggerated, whereas the potential negative effects are typically ignored or underemphasized. Hence, it is critical to understand and properly manage firm capabilities and competencies to minimize and avoid risky investments in innovation.

Innovation supports management by introducing new services, products, and improvements in quality [5]; thus, innovation has significantly influenced business outcomes by enhancing the competitiveness and sustainability of businesses [20]. Nonetheless, Hjalager [9] noticed a gap between innovation and sustainable business; thus, the manner in which innovation assists sustainable business management must be investigated to gain greater and deeper insights for hotel managers. In addition, sustainable business management capabilities and competencies (SBMCC) must be identified and utilized to promote innovation efficiency and effectiveness and to enable firms to enjoy and foster a sustainable business [21,22]. This study suggests that technology capabilities play an important role in innovation, and the readiness to upgrade and integrate new technology can enable innovation for product and service development, marketing, and even for management processes. Moreover, networking and social capabilities were determined to be important in gathering and sharing knowledge, which assists a firm's innovation output by enabling innovative collaboration [23-25]. 
Certain measures play a significant role in defining and illuminating SBMCC because the complex, limited, and diverse information available regarding SBMCC has led to incomplete and vague descriptions of the notion. Moreover, these measures have provided qualitative information and linguistic descriptions, as well as incomplete information, from firms. In particular, imprecise information has resulted because of situations in which there are insufficient data and the data pattern remains unknown [26,27]. Therefore, this evaluation system would be well-served by applying a classic grey system. In addition, this study must utilize experts' subjective opinions, assigning them relative weights, and combining them with grey relation analysis (GRA) to evaluate and understand the important aspects and criteria of particular firms. To identify the relative weights of the proposed aspects and criteria is an important part of this approach, and this study applies interval-valued triangular fuzzy numbers (IVTFN) for the subjective approaches. To overcome the shortcomings discussed above, this study proposes IVTFN and GRA together to shed additional light on SBMCC in firms. Finally, achieving a sustainable business requires capabilities and competencies to understand the innovation activities. Hence, the objective of this study is to investigate the contributions of innovation criteria to SBMCC under uncertainty and to demonstrate the effects on SBMCC when there is incomplete information. Accordingly, this study is organized as follows. First, innovation and its relationship with SBMCC are presented. In Section 2, the theory adopted is discussed. Section 3 presents the methods. The results are discussed in Section 4. Section 5 presents the theoretical and managerial implications. The last section concludes and discusses the limitations of this study.

\section{Literature Review}

This section reviews the definitions and the role of innovation in SBMCC. The subsection review emphasizes the following specific areas: innovation, SBMCC, the proposed method, and the development of the proposed measures.

\subsection{Innovation}

Van Kleef and Roome [21] defined "innovation as the process of discovery and development that generates new products, production processes, organizations, technology, and institutional and systemic arrangements". This definition includes employing ideas, knowledge, and technology in a manner that enables firms to significantly improve performance. Onsel et al. [28] indicated that innovation is not necessarily related to problem-solving but is instead typically related to improving competitiveness and economic success - and it is frequently spurred by technology. The previous literature distinguished the different types of innovation as technology, process, product and service, management, operations, and organization $[29,30]$.

Innovation was viewed as supporting businesses in a variety of ways, such as in product and service innovation; technological innovation can increase the efficiency and effectiveness of a firm's operations, whereas organizational innovation can promote growth in productivity and competitiveness by encouraging the application of new technologies [31]. Several studies have indicated that innovation is directly or indirectly linked to the sustainable business management of a firm [32,33]. Hence, the contributions of innovation to sustainable business management might be implemented by utilizing and developing firm capabilities and competencies [21]. However, capability and competency problems also have affected 
innovation activities. Van de Ven and Engleman [34] noted the problem of managing networking and social capabilities; innovative ideas arise from different areas inside or outside a firm, and multiple functions and resources within a corporation are necessary to transform an innovative idea into reality.

\subsection{Innovation for Sustainable Business Management Capabilities and Competencies}

Van Kleef and Roome [21] defined sustainable business management as "the management of sustainable business that recognizes its embeddedness in social, environmental and economic systems and focuses on management and relationships to meet the environmental, social, and economic requirements of the many different stakeholders in its networks". Recently, innovation for sustainable business management has received an increasing amount of attention from management [35], as innovation is increasingly recognized as an important means to contribute to sustainability [36,37]. However, people and firms tend to focus on and take advantage of existing practices rather than exploring new ideas, needs, and opportunities for innovation; thus, it is difficult to encourage innovation in these cases [34]. Hence, there is an essential need for firms to explore the insights of capabilities and competencies to drive innovation.

Dutta et al. [38] considered capability as the ability to transform resources into objectives, which relates to performing a set of synchronizing tasks and utilizing resources to attain a particular result. These results offer an individual or an entire organization the ability to construct the building blocks of business competencies [21]. Thus, competencies are combinations of knowledge, experience, and productive attributes, in addition to being accurate combinations of functional and technical skills to perform the tasks, solve problems, and operate effectively under uncertainty [39-42]. Competencies are also represented in the entirety of the firm, which contains the contribution of employees, alignments within the firm, and the value added to customers and stakeholders. These attributes allow firms to create new products and services while adapting to changing circumstances faster than competitors and thus enhancing their competiveness [5].

This study extends the definition of SBMCC from van Kleef and Roome [21]: "Sustainable business management builds embeddedness capabilities and competencies by addressing the social, environmental and economic systems and focuses on innovation and relationships to meet the different stakeholder requirements in its networks". Ensuring that innovation competencies are present in the key roles within a firm and that these competencies are used to build internal capabilities at all levels is also essential to the pursuit of business sustainability [42-44]. In particular, innovation yields benefits to SBMCC by leveraging those capabilities and competencies that enable firms to build cost efficiencies and operational effectiveness to compete in a sustainable business environment. To enhance the understanding in this field, this study proposes five aspects by which to identify SBMCC: (1) technological capabilities; (2) networking and social capabilities; (3) learning and developing capabilities; (4) competencies for systemic thinking; and (5) capabilities for integrating business, environmental/social problems, perspectives and information.

\subsection{Proposed Method}

Previous studies have focused on applying classical statistical methods in addressing sustainable business management $[45,46]$. Few studies have considered SBMCC as a multiple criteria problem to identify its importance by adopting linguistic variables and providing sufficient results. Within the 
identification, linguistic variables rely on expert opinions and contain vagueness, imprecision, and uncertainties [47]. Hence, IVTFN is proposed in this study to overcome these situations and to convert the linguistic variables into quantitative evaluations. Therefore, the computation of the geometric mean is taken as the membership degree to derive the statistical unbiased effect and avoid the impact of extreme values. The advantage of this method is the simplicity that results from all the expert opinions being contained in one investigation and that aggregates the evaluations from diverse perspectives. Once the fuzzy decision matrix is obtained, the GRA must arrange to address the incomplete system information.

GRA was developed by Deng [48] to identify the relation between two sequences. This method is suggested as an instrument for solving the multiple criteria problem, which enables us to find solutions from a finite set of aspects and criteria under incomplete information [26,27,49]. The basic principle is that if a comparability sequence translated from a criterion has the highest grey relational grade among the reference sequence, it represents the best choice. All the criteria can be ranked upon the grey relational grade, which will help the decision-maker in handling a multi-criteria decision-making problem. This study integrated IVTFN and GRA to illuminate the relationship between innovations and SBMCC. The criteria are formed as linguistic variables and expressed in IVTFN, then GRA is used to determine the ranking of aspects and criteria under incomplete information [50].

\subsection{Proposed SBMCC Measures}

The previous literature has been used to identify the capabilities and competencies required to innovate for competitive purposes in sustainable business management [21,42-44]. However, previous studies in the field of innovation have discussed these capabilities and competencies separately, which include systems thinking; learning and developing; networking and social capabilities; integrating business, environmental, information and social problems; and technology (as shown in Table 1). A comprehensive study is required to aggregate all as a whole to understand how these capabilities and competencies can lead to innovation for sustainable business management. Hence, the multi-criteria approach is adopted in this study to derive the significant implications resulting from our approach.

Systems thinking is a new concept in addressing competencies; accordingly, product invention, creativity, processes and/or business models require firm personnel to learn from experience in terms of both practice and theory to contribute to job performance and new firm projects $[21,51,52]$. Moreover, systems thinking can utilize multi-theories and techniques to build holistic, contingent perspectives and practices [53-55]. Independent thinking creates diversity and the potential to contribute to organizational learning and to address complexity [52,56] - the individual is the building block in the development of diverse skills and competencies [21,57]. However, teammates who have developed their own specific abilities and inventive strategies may increase the possibility of bringing ideas together to solve problems [58]. 
Table 1. Proposed innovation measures for sustainable business management capabilities and competencies.

\begin{tabular}{|c|c|c|}
\hline Aspects & & Criteria \\
\hline \multirow{6}{*}{$\begin{array}{l}\text { Competencies } \\
\text { for systems } \\
\text { thinking (AS1) }\end{array}$} & $\mathrm{C} 1$ & The competency to think independently \\
\hline & $\mathrm{C} 2$ & $\begin{array}{l}\text { Expertise in organization design will be a critical skill- }- \text { how to analyze, } \\
\text { modify and simulate the behavior of complex human systems }\end{array}$ \\
\hline & $\mathrm{C} 3$ & A powerful tool to facilitate both individual and organizational learning \\
\hline & $\mathrm{C} 4$ & Product life-cycle management systems offer core innovation competencies \\
\hline & $\mathrm{C} 5$ & The competency to think inventively \\
\hline & C6 & Grasping manufacturing cycle time will improve on-time delivery, product quality and cost \\
\hline \multirow{6}{*}{$\begin{array}{l}\text { Networking and } \\
\text { social capabilities } \\
\text { (AS2) }\end{array}$} & $\mathrm{C} 7$ & To gather and review the alternative strategies of each stakeholder \\
\hline & $\mathrm{C} 8$ & To build trust, a shared vision and agreement on basic values \\
\hline & C9 & $\begin{array}{l}\text { To develop social relations with unfamiliar actors inside or outside the organization } \\
\text { for information gathering, experimentation and negotiation purposes }\end{array}$ \\
\hline & $\mathrm{C} 10$ & $\begin{array}{l}\text { To involve many stakeholders from the start (e.g., local communities, } \\
\text { regional, state or national stakeholders, supply chain partners, and buyers) }\end{array}$ \\
\hline & $\mathrm{C} 11$ & To co-operate with universities/research institutes, to develop brand new ideas \\
\hline & $\mathrm{C} 12$ & $\begin{array}{l}\text { To participate in industrial networks such as industrial } \\
\text { associations, standard organizations and industrial forums }\end{array}$ \\
\hline \multirow{6}{*}{$\begin{array}{c}\text { Technology } \\
\text { capabilities (AS3) }\end{array}$} & $\mathrm{C} 13$ & $\begin{array}{l}\text { New approaches to developing knowledge for performing management } \\
\text { functions and new processes that produce changes in } \\
\text { the organization's strategy, structure, administrative procedures, and systems }\end{array}$ \\
\hline & $\mathrm{C} 14$ & To upgrade and integrate technology capabilities, new product development and marketing \\
\hline & $\mathrm{C} 15$ & Using existing technology \\
\hline & $\mathrm{C} 16$ & $\begin{array}{l}\text { Adoption of electronic data processing for a variety of internal information storage, } \\
\text { retrieval and analytical purposes, indirectly related to the basic work activity of } \\
\text { the firm and more immediately related to its management }\end{array}$ \\
\hline & $\mathrm{C} 17$ & Quantum leaps in performance \\
\hline & $\mathrm{C} 18$ & To define technology that could help \\
\hline \multirow{6}{*}{$\begin{array}{l}\text { Capabilities for } \\
\text { learning and } \\
\text { developing (AS4) }\end{array}$} & $\mathrm{C} 19$ & To learn and translate learning into action \\
\hline & $\mathrm{C} 20$ & Typically adopt new ideas and develop them as reliable products \\
\hline & $\mathrm{C} 21$ & $\begin{array}{l}\text { To effectively address the requirements, values, assumptions } \\
\text { and cultures of various interacting network actors }\end{array}$ \\
\hline & $\mathrm{C} 22$ & $\begin{array}{l}\text { To develop insights and observe what local community does, how they think, } \\
\text { what they need and want }\end{array}$ \\
\hline & $\mathrm{C} 23$ & To encourage cross-functional learning and fertilization \\
\hline & $\mathrm{C} 24$ & To successfully understand and execute innovation activities with the network \\
\hline \multirow{6}{*}{$\begin{array}{c}\text { Capabilities for } \\
\text { integrating business, } \\
\text { environmental, } \\
\text { information and } \\
\text { social problems. } \\
\text { (AS5) }\end{array}$} & $\mathrm{C} 25$ & To integrate the perspectives and knowledge of different actors in the network \\
\hline & $\mathrm{C} 26$ & To integrate traditional criteria of efficiency with eco- and social-efficiency and effectiveness \\
\hline & $\mathrm{C} 27$ & To integrate one or two socio-environmental indicators into a single perspective \\
\hline & $\mathrm{C} 28$ & To apply the knowledge gained in previous projects to new projects \\
\hline & $\mathrm{C} 29$ & $\begin{array}{l}\text { To integrate differences in information processing and decision styles, } \\
\text { to address differences in the width of focus and the desired degree of maximization of the result }\end{array}$ \\
\hline & $\mathrm{C} 30$ & To identify the business problem and constraints (time, resources, market) \\
\hline
\end{tabular}


In the case of learning and developing, different actors in the networks provide greater potential for learning and are necessary to evoke the potential solutions to solve complex issues [63,64]. These actors are internal and external to the firm and develop insights and observe what the local community does, how individual members think, and what they need and want; these actors understand and execute innovation activities within the network by developing knowledge and forming new innovation networks [61,63]. Although all the firms aim to reach this level, most encounter difficulties in the basic practice that requires collaboration in various business and management functions, such as marketing affiliations and certification schemes aligning to promote environmental awareness and education as well as new product development and innovation [65].

For a firm, competitive advantage not only is dependent on research and development but also is enhanced by potential technology $[60,66]$. In reality, most of the firm is unable to have up-to-date technology developed in-house because of the increasingly complex nature of technology and short product life cycles. If a firm wants to remain competitive in the market, it must quickly integrate, adopt, and upgrade the diversity of its external and internal information storage, retrieval, and analytical tools that relate to basic work activities — in addition to business and management functions - with external technologies and on-time product launches $[21,60]$. Therefore, searching for the internal factors that encourage technological innovation capabilities might augment the understanding of innovative processes in the firm $[67,68]$.

Some sustainable actions involve collective problem solving, system development, technological progress, and other innovations that are identified with achieving business goals. These stages can be presented in the open processes of innovation and can input the implementation into a broad range of stakeholders. Hence, the social networks offer a platform for individuals and stakeholders in a community to reference, gather, and exchange ideas, information, experimentation, and negotiation [69]. Although these platforms can be designed for searching opportunities, it remains insufficient to discuss the sustainability issues currently $[21,65]$. Thus, firms require a strategy that is compatible with integrating into these platforms and with these stakeholders, that creates competitive advantages such as cost reductions and favorable future market positions, and that benefits the firm by enabling it to acquire increased legitimacy through collaborations with external stakeholders in a community [70].

Dyllick and Hockerts [71] have suggested that sustainable business management requires the concept of efficiency (the economic value added in relation to the aggregate resources used) to be extended to eco-efficiency and socio-efficiency (the economic value added in relation to the aggregated ecological and social impacts, respectively). In addition, concepts such as eco-effectiveness and socio-effectiveness have been suggested as ways to address the absolute thresholds in social and ecological sustainability [72]. The European Commission [73] has suggested that efficiency might be complemented or replaced by the notion of sufficiency. Deciding upon the size and character of social and environmental thresholds and determining sufficiency criteria are understood to require inputs from actors outside of firms as units of production $[65,74]$.

\section{Method}

GRA was initially proposed by Deng [75] to overcome the insufficient information among systems. In addition, IVTFN was used to assist decision-makers in the precise evaluation of subjects. This study 
integrates GRA and IVTFN to explore the important aspects and criteria for building sustainability under uncertainty. The method and proposed analytical steps are described below.

\subsection{Interval-Valued Triangular Fuzzy Numbers with Grey Relational Analysis}

Consider a decision-making problem with $i$ aspects and $j$ criteria; then, let $\alpha=\left\{\alpha_{1}, \alpha_{2}, \cdots, \alpha_{i}\right\}$ and $\beta=\left\{\beta_{1}, \beta_{2}, \cdots, \beta_{\mathrm{j}}\right\}$ be a finite set of feasible aspects and criteria, respectively. Although the weightage of criteria $v=\left(v_{1}, v_{2}, \cdots v_{j}\right)$ is unknown, it must satisfy $v_{j} \geq 0, j=1,2, \cdots \mathrm{n}, \sum_{\mathrm{j}=1}^{\mathrm{n}} v_{j}=1$. Assume that the performance $\beta_{j}$ related to aspect $\alpha_{i}$ is defined as $s_{\mathrm{ij}}$; then, the decision matrix can be presented by $S=\left[s_{i j}\right]_{\mathrm{m} \times \mathrm{n}}$. According to the illustration of Figure 1, $s_{i j}$ can be rewritten as IVTFN s' $=\left\{\begin{array}{l}\left(\mathrm{s}_{1}, \mathrm{~s}_{2}, \mathrm{~s}_{3}\right) \\ \left(\tilde{\mathrm{s}}_{1}, \mathrm{~s}_{2}, \widetilde{\mathrm{s}}_{3}\right)\end{array}\right.$ and is also expressed as $s^{\prime}=\left[\left(s_{1}, \widetilde{s}_{1}\right) ; s_{2} ;\left(\widetilde{s}_{3}, s_{3}\right)\right]$.

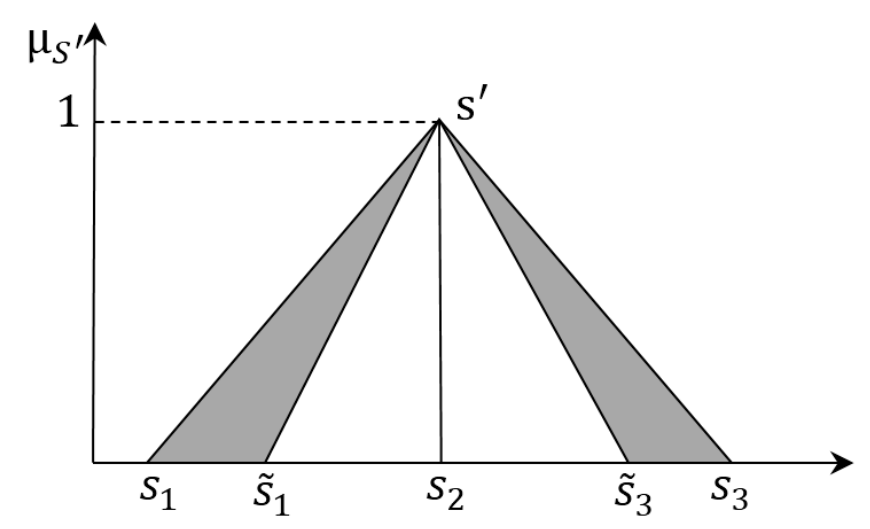

Figure 1. An interval-valued triangular fuzzy number.

The proposed IVTFN-GRA method is associated with an unknown weightage vector and calculated by the following procedures. First, normalize the decision matrix $S$, which consists of $s_{i j}^{\prime}=\left[\left[\left(x_{i j}, \tilde{x}_{i j}\right) ; y_{i j} ;\left(\tilde{z}_{i j}, z_{i j}\right)\right]\right]$, as indicated in Table 2. If there are $k$ experts in the expert group, the responses must be calculated in $s_{i j}^{\prime}=\frac{1}{k}\left(s_{i j}^{\prime 1}+s_{i j}^{\prime 2}+s_{i j}^{\prime 3}+\cdots s_{i j}^{\prime k}\right)=\frac{1}{k} \sum_{1}^{k} s_{i j}^{\prime n}$. In addition, the responses must normalize into performance rating matrix $S^{*}=\left[s_{i j}^{*}\right]_{m \times n}$ as below:

$$
\begin{aligned}
& {\left[s_{i j}^{B}\right]_{m \times n}=\left[\left(\frac{x_{i j}}{z_{j}^{+}}, \frac{\tilde{x}_{i j}}{z_{j}^{+}}\right) ; \frac{y_{i j}}{z_{j}^{+}} ;\left(\frac{\tilde{z}_{i j}}{z_{j}^{+}}, \frac{z_{i j}}{z_{j}^{+}}\right)\right]_{m \times n}, i=1,2, \cdots, m ; j=1,2, \cdots, n, \text { for } j \in B} \\
& {\left[s_{i j}^{C}\right]_{m \times n}=\left[\left(\frac{x_{j}^{-}}{z_{i j}}, \frac{x_{j}^{-}}{\tilde{z}_{i j}}\right) ; \frac{x_{j}^{-}}{y_{i j}} ;\left(\frac{x_{j}^{-}}{\tilde{x}_{i j}}, \frac{x_{j}^{-}}{x_{i j}}\right)\right]_{m \times n}, i=1,2, \cdots, m ; j=1,2, \cdots, n, \text { for } j \in C}
\end{aligned}
$$

where $z_{j}^{+}=\max _{i}\left\{z_{i j}, i=1 \cdots m\right\}, x_{j}^{-}=\min _{i}\left\{x_{i j}, i=1 \cdots m\right\}$, and $B$ and $C$ are the sets of benefit and cost criteria. 
Table 2. Definitions of linguistic variables for the importance ratings.

\begin{tabular}{cc}
\hline Linguistic Variables & Interval-Valued TFNs \\
\hline Very unimportant $(\mathrm{VU})$ & {$[(0,0) ; 0 ;(0.1,0.15)]$} \\
Unimportant $(\mathrm{U})$ & {$[(0,0.05) ; 0.1 ;(0.25,0.35)]$} \\
Medium unimportant $(\mathrm{MU})$ & {$[(0,0.15) ; 0.3 ;(0.45,0.55)]$} \\
Medium $(\mathrm{M})$ & {$[(0.25,0.35) ; 0.5 ;(0.65,0.75)]$} \\
Medium important (MI) & {$[(0.45,0.55) ; 0.7 ;(0.8,0.95)]$} \\
Important (I) & {$[(0.55,0.75) ; 0.9 ;(0.95,1)]$} \\
Very important (VI) & {$[(0.85,0.95) ; 1 ;(1,1)]$} \\
\hline
\end{tabular}

Second, determine the reference series $R_{0}=\left(r_{01}, r_{02}, \cdots, r_{0 n}\right)$, which is supposed to be 1 as in the case of the reference value in the decision-making problem. Hence, the reference series can be rewritten as $R_{0}=([(1,1) ; 1 ;(1,1)],[(1,1) ; 1 ;(1,1)], \cdots,[(1,1) ; 1 ;(1,1)])$. Once the reference series is obtained, the following computation must identify the gap between the reference value and each comparison value. The computation can use the following equations:

$$
\left\{\begin{array}{l}
\vartheta_{i j}^{(B)}=\sqrt{\frac{1}{3}\left[\left(\frac{\tilde{x}_{i j}}{z_{j}^{+}}-1\right)^{2}+\left(\frac{y_{i j}}{z_{j}^{+}}\right)^{2}+\left(\frac{z_{i j}}{z_{j}^{+}}-1\right)^{2}\right]} \\
\vartheta_{i j}^{(\bar{B})}=\sqrt{\frac{1}{3}\left[\left(\frac{x_{i j}}{z_{j}^{+}}-1\right)^{2}+\left(\frac{y_{i j}}{z_{j}^{+}}\right)^{2}+\left(\frac{\tilde{z}_{i j}}{z_{j}^{+}}-1\right)^{2}\right]} \\
\vartheta_{i j}^{(C)}=\sqrt{\frac{1}{3}\left[\left(\frac{x_{j}^{-}}{\tilde{z}_{i j}}-1\right)^{2}+\left(\frac{x_{j}^{-}}{y_{i j}}\right)^{2}+\left(\frac{x_{j}^{-}}{x_{i j}}-1\right)^{2}\right]} \\
\vartheta_{i j}^{(\bar{C})}=\sqrt{\frac{1}{3}\left[\left(\frac{x_{j}^{-}}{z_{i j}}-1\right)^{2}+\left(\frac{x_{j}^{-}}{y_{i j}}\right)^{2}+\left(\frac{x_{j}^{-}}{\tilde{x}_{i j}}-1\right)^{2}\right]}
\end{array}\right.
$$

The gap can be simplified as an interval value $\vartheta_{i j}^{*}=\left[\hat{\vartheta}_{i j}, \check{\vartheta}_{i j}\right]$, where $\hat{\vartheta}_{i j}=\vartheta_{i j}^{(B)}-\vartheta_{i j}^{(\bar{B})}$ and $\breve{\vartheta}_{i j}=\vartheta_{i j}^{(C)}-\vartheta_{i j}^{(\bar{C})}$. Because some information is lost during the computation process, the interval value must convert into a crisp value immediately. Simultaneously, the maximum $\left[\rho^{\prime}, \gamma^{\prime}\right]$ and minimum $\left[\rho^{-}, \gamma^{-}\right]$can be gathered from the crisp value, as below:

$$
\left\{\begin{array}{l}
{\left[\rho^{\prime}, \gamma^{\prime}\right]=\left[\max _{i j} \hat{\vartheta}_{i j}, \max _{i j} \breve{\vartheta}_{i j}\right]} \\
{\left[\rho^{-}, \gamma^{-}\right]=\left[\min _{i j} \hat{\vartheta}_{i j}, \min _{i j} \breve{\vartheta}_{i j}\right]}
\end{array}, i=1,2, \cdots, m ; j=1,2, \cdots, n\right.
$$

Third, assuming $\tau$ is 0.5 and using the following equations to generate the grey relational coefficient:

$$
\left\{\begin{array}{l}
\varepsilon_{i j}^{\prime}=\rho^{-}+\tau \rho^{\prime} / \hat{\vartheta}_{i j}+\tau \rho^{\prime} \\
\varepsilon_{i j}^{*}=\gamma^{-}+\tau \gamma^{\prime} / \check{\vartheta}_{i j}+\tau \gamma^{\prime}
\end{array}, i=1,2, \cdots, m ; j=1,2, \cdots, n\right.
$$

To obtain the grey relational grade, Zhang et al. [76] suggested normalizing the data by adopting weightage vector $v_{j}$ :

$$
v_{j}=\sum_{i=1}^{m}\left(\varepsilon_{i j}^{\prime}+\varepsilon_{i j}^{*}\right) / \sum_{i=1}^{m} \sum_{j=1}^{n}\left(\varepsilon_{i j}^{\prime}+\varepsilon_{i j}^{*}\right), j=1,2, \cdots, n
$$


When the weightage vector $v=\left(v_{1}, v_{2}, \cdots v_{n}\right)$ is attained, the grey relational grade can be expressed as an interval value $D_{i}=\left[\bar{d}_{i}, \tilde{d}_{i}\right]$ :

$$
\bar{d}_{i}=\sum_{j=1}^{n} v_{j} \varepsilon_{i j}^{\prime}, \tilde{d}_{i}=\sum_{j=1}^{n} v_{j} \varepsilon_{i j}^{*}, i=1,2 \cdots, m
$$

Therefore, the grey relational grade must comply with the likelihood properties to transfer the interval value to a likelihood matrix. Li et al. [77] presented the following helpful likelihood properties:

$$
\left\{\begin{array}{c}
0 \leq \mu(x \geq y) \leq 1 \\
\mu(x \geq y)+\mu(y \geq x)=1 ; \\
\mu(x \geq y)+\mu(y \geq x)=0.5 \text { if } \mu(x \geq y)=\mu(y \geq x) \\
\mu(x \geq y)=0 \text { if } x^{+} \leq y^{-} ; \\
\text {For any interval numbers } x, y \text { and } z, \mu(x \geq z)=\mu(y \geq z) \text { if } x \geq y
\end{array}\right.
$$

Finally, transform the grey relational grade into likelihood relations. The likelihood relation can be denoted as $\alpha_{o} \geqslant \alpha_{p}$, which means "criteria $\alpha_{o}$ being not inferior to $\alpha_{p}$ ". Li et al. [77] indicated that the likelihood relation of $\alpha_{o} \geqslant \alpha_{p}$ is evaluated by $G_{o} \geqslant G_{p}$, where $G_{o}$ and $G_{p}$ are corresponding grey relational grade interval numbers of criteria $\alpha_{o}$ and $\alpha_{p}$ in $\alpha$. The transforming process applies the equation as below:

$$
\rho\left(\alpha_{o} \geqslant \alpha_{p}\right)=\rho\left(D_{o} \geqslant D_{p}\right)=\max \left\{1-\max \left\{\left(\tilde{d}_{p}-\bar{d}_{o}\right) /\left(\varphi\left(D_{o}\right)+\varphi\left(D_{p}\right)\right), 0\right\}, 0\right\}
$$

where $D_{o}=\left[\bar{d}_{o}, \tilde{d}_{o}\right], D_{p}=\left[\bar{d}_{p}, \tilde{d}_{p}\right], \varphi\left(D_{o}\right)=\tilde{d}_{o}-\bar{d}_{o}, \varphi\left(D_{p}\right)=\tilde{d}_{p}-\bar{d}_{p}$.

The likelihood relations can be arranged into the likelihood matrix as $\tilde{S}$ :

$$
\tilde{S}=\left(\rho_{o p}\right)_{m \times m}=\begin{array}{cccc}
\alpha_{1} & \alpha_{2} & \cdots & \alpha_{m} \\
\alpha_{1} \\
\alpha_{2} \\
\vdots \\
\alpha_{\mathrm{m}}
\end{array} \quad\left(\begin{array}{cccc}
\rho_{11} & \rho_{12} & \cdots & \rho_{1 \mathrm{~m}} \\
\rho_{21} & \rho_{22} & \cdots & \rho_{2 \mathrm{~m}} \\
\vdots & \vdots & & \vdots \\
\rho_{\mathrm{m} 1} & \rho_{\mathrm{m} 1} & \cdots & \rho_{\mathrm{mm}}
\end{array}\right)
$$

where $\rho_{o p}=\rho\left(\alpha_{o} \geqslant \alpha_{p}\right)(o, p=1,2, \cdots, m)$ for criteria $\alpha_{o}$ and $\alpha_{p}$. Consequently, the $\tilde{S}$ is a complementary judgment matrix, and the ranking weightage can be sorted from the eigenvector $E_{i}(i=$ $1,2, \cdots m)$. The larger value of $E_{i}$ represents the greater importance of criteria $\alpha_{\mathrm{i}}(i=1,2, \cdots m)[76]$.

\subsection{Proposed Analytical Step}

This study adopts IVTFN and GRA to evaluate five aspects and 30 criteria. The objective is to determine the priority ranking in innovation for SBMCC. The expert group followed the proposed solution with a four-step procedure. The analysis procedures are explained as follows:

(1) Identification of the evaluating aspects and criteria. This step collects group knowledge and experiences from the expert committee to evaluate the importance of SBMCC. The committee develops the aspects and criteria — and the survey instruments - to establish a set of aspects and criteria for evaluation. The cluster of aspects and criteria has the nature of complicated relationships. 
(2) Computation of the range between the experts' decisions related to each criterion. In the beginning of this step, this study used interval-valued triangular fuzzy numbers (as indicated in Table 2) to normalize the decision matrix into an important rating. If the expert committee has different opinions about the decision, the average scores can eliminate the situation. Benefit and cost can be found by following Equations (1) and (2). Next, calculating the distance is required to contract with the reference value and transfer to the interval value by Equation (3). However, some information has been lost during the computation, so the interval values must be expressed as crisp values immediately, as in Equation (4).

(3) Once the crisp value is obtained, the grey relational coefficient must be generated by Equation (5). Before converting the grey relational coefficient to the grey relational grade, the weightage vector must be obtained through Equation (6) first and then Equation (7) is used to finish the transformation. Consequently, the grey relational grade must associate with likelihood properties as in Equation (8) to calculate the likelihood relations.

(4) Then, adopt Equation (9) to gather all likelihood relations and arrange them into a matrix by adopting Equation (10). The matrix is decomposed using MATLAB 10 to acquire the eigenvectors for each of the criteria. The largest eigenvector has the greatest effect on SBMCC.

\section{Results}

This section presents an overview of Taiwanese hotels and data collection methods and is divided into two subsections: industrial background and analytical results.

\subsection{Industrial Background}

The tourism industry continues to expand and diversify and has become one of the largest and fastest growing economic sectors in the world [78]. In Taiwan, the demand for recreational travel has increased rapidly as the number of foreign visitors has increased. The tourism industry not only brings in substantial foreign exchange income but also provides job opportunities. The Tourism Bureau of the Ministry of Transportation and Communications of Taiwan 2014 has announced new directions for the Taiwan tourism industry. Hopefully, the number of foreign tourists visiting Taiwan will continue to increase, thus benefiting the hotel industry. Additionally, several international hotel chains are racing to boost their presence in response to the booming tourism industry due to globalization. This intensifies the competition in the hospitality industries, particularly in hotel firms.

To assist firms in the hotel industry in this competitive environment and to guarantee sustainable growth, this study has been conducted to obtain a better understanding of the contribution of innovation to sustainable business, thus providing several managerial insights related to SBMCC. This study adopted GRA and IVTFN to evaluate the importance of certain aspects and criteria. Questionnaires were created based on interviews with executive management and industrial experts to collect the aspects and criteria. The analysis outlined in the following section describes the process followed by the hotels in Taiwan and the recommendations given to them. 


\subsection{The Empirical Results}

Gathering the respondent opinions ensures that clear relationships are determined in the evaluation of aspects and criteria; it also confirms the information that is relevant to representing the SBMCC by consulting the expert committee. Each proposed aspect and criterion in Table 1 prompted several arguments and discussions among the members of the expert committee related to enhancing the study's validity and reliability. Once the experts raised an argument or debate, interviewing was adopted for further clarification. To overcome problematic linguistic preferences and complicated interactions in the respondent feedback, a hybrid method was designed integrating IVTFN and GRA.

(1) Table 3 presents the preference information of the experts' responses. The preference information must convert into IVTFNs based on Table 1; the average IVTFNs are displayed in Table 4. Tables 5 and 6 display the benefit and cost criteria matrices generated by Equations (1) and (2). 
Table 3. Experts' responses.

\begin{tabular}{|c|c|c|c|c|c|c|c|c|c|c|c|c|c|c|c|c|c|c|c|c|c|c|c|c|c|c|c|c|c|c|c|c|c|c|c|}
\hline & \multicolumn{6}{|c|}{ Expert 1} & \multicolumn{4}{|c|}{ Expert 2} & \multicolumn{5}{|c|}{ Expert 3} & \multicolumn{5}{|c|}{ Expert 4} & \multicolumn{5}{|c|}{ Expert 5} & \multicolumn{5}{|c|}{ Expert 6} & \multicolumn{5}{|c|}{ Expert 7} \\
\hline & AS1 & AS2 & & AS4 & & & & AS3 & & & & & AS3 & AS4 & & & & AS3 & AS4 & & & AS2 & AS3 & S4 & AS5 & AS1 & AS2 & AS3 & AS4 & AS5 & AS1 & AS2 & AS3 & AS4 & AS5 \\
\hline $\mathrm{C} 1$ & $M$ & $\mathrm{I}$ & MI & $\mathrm{M}$ & MU & M & VI & MI & H & M & VU & I & MI & $\mathrm{U}$ & $\mathrm{U}$ & $\mathrm{M}$ & VI & MI & M & M & $M$ & VI & I & $M$ & $M$ & $\mathrm{U}$ & $\mathrm{I}$ & I & $\mathrm{U}$ & $\mathrm{U}$ & I & VI & VI & MU & M \\
\hline $\mathrm{C} 2$ & MU & I & MI & MU & U & M & VI & MI & & $\mathrm{U}$ & M & VI & MI & M & M & VU & & I & I & M & M & 1 & 1 & $\mathrm{U}$ & VU & $M$ & 1 & 1 & $M$ & $\mathrm{U}$ & M & M & VI & U & $\mathrm{U}$ \\
\hline $\mathrm{C} 3$ & $\mathrm{U}$ & VI & I & MU & MU & $\mathrm{U}$ & I & VI & $\mathrm{U}$ & $\mathrm{U}$ & M & VI & I & M & M & M & VI & VI & M & M & U & I & VI & U & M & U & 1 & MI & M & M & $\mathrm{U}$ & VI & VI & MU & M \\
\hline $\mathrm{C} 4$ & $\mathrm{U}$ & I & I & VU & MU & $\mathrm{U}$ & I & I & MU & $\mathrm{U}$ & M & I & M & M & U & M & I & $\mathrm{N}$ & M & VU & 1 & 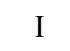 & M & & $\mathrm{N}$ & M & $4 \mathrm{~L}$ & $M$ & VU & $\mathrm{V}$ & I & M & M & VU & VU \\
\hline $\mathrm{C} 5$ & $\mathrm{U}$ & I & VI & MU & $\mathrm{M}$ & M & I & I & M & MU & $\mathrm{U}$ & MI & MI & MU & M & M & VI & VI & $\mathrm{M}$ & I & $\mathrm{U}$ & VI & VI & $\mathrm{U}$ & $\mathrm{U}$ & $\mathrm{U}$ & I & VI & $\mathrm{U}$ & $\mathrm{U}$ & $\mathrm{U}$ & VI & VI & I & $\mathrm{MU}$ \\
\hline C6 & $\mathrm{U}$ & VI & VI & MU & MU & VU & VI & I & I & $\mathrm{U}$ & VU & VI & VI & $\mathrm{U}$ & 1010 & $\mathrm{M}$ & 1 & I & I & $\mathrm{M}$ & $\mathrm{U}$ & VI & VI & $\mathrm{M}$ & $\mathrm{U}$ & $\mathrm{U}$ & I & MI & $\mathrm{M}$ & $\mathrm{M}$ & $\mathrm{MU}$ & VI & VI & MU & $\mathrm{M}$ \\
\hline $\mathrm{C} 7$ & $\mathrm{M}$ & I & I & MU & $\mathrm{U}$ & $\mathrm{U}$ & I & I & VI & MU & VU & MI & I & MU & $\mathrm{MU}$ & $\mathrm{M}$ & VI & I & M & M & $\mathrm{U}$ & I & I & M & M & $\mathrm{U}$ & I & I & $\mathrm{U}$ & $\mathrm{U}$ & I & M & VI & MU & $M$ \\
\hline C8 & $\mathrm{U}$ & VI & I & $\mathrm{M}$ & $\mathrm{MU}$ & VU & VI & VI & I & $\mathrm{M}$ & $\mathrm{M}$ & MI & MI & MU & $\mathrm{M}$ & $\mathrm{M}$ & I & VI & I & M & $\mathrm{U}$ & I & VI & $\mathrm{M}$ & $\mathrm{U}$ & $\mathrm{U}$ & I & I & M & $\mathrm{U}$ & $\mathrm{U}$ & VI & I & I & I \\
\hline C9 & I & VI & VI & I & I & I & VI & VI & I & I & I & VI & VI & I & I & I & VI & VI & MI & I & I & VI & VI & 1 & I & I & VI & VI & I & I & I & VI & VI & I & I \\
\hline $\mathrm{C} 10$ & MU & VI & I & $\mathrm{U}$ & VU & $\mathrm{M}$ & I & $\mathrm{M}$ & VU & $\mathrm{M}$ & VU & I & MI & MU & $\mathrm{U}$ & $\mathrm{M}$ & I & I & I & $\mathrm{M}$ & M & I & I & $\mathrm{M}$ & $\mathrm{U}$ & $\mathrm{U}$ & I & I & $\mathrm{U}$ & $\mathrm{U}$ & I & VI & I & $\mathrm{M}$ & $\mathrm{MU}$ \\
\hline C11 & $\mathrm{U}$ & VI & I & $\mathrm{U}$ & $\mathrm{U}$ & VU & VI & VI & $\mathrm{U}$ & $\mathrm{U}$ & VU & MI & MI & MU & $\mathrm{M}$ & $\mathrm{M}$ & I & VI & $\mathrm{M}$ & $\mathrm{M}$ & $\mathrm{U}$ & VI & VI & $\mathrm{M}$ & $\mathrm{M}$ & $\mathrm{U}$ & I & I & $\mathrm{M}$ & $\mathrm{U}$ & $\mathrm{U}$ & VI & MI & MU & I \\
\hline $\mathrm{C} 12$ & $\mathrm{M}$ & I & I & $\mathrm{U}$ & $\mathrm{M}$ & vo & VI & VI & MU & MU & $\mathrm{M}$ & VI & VI & MU & $\mathrm{M}$ & $\mathrm{M}$ & I & I & $\mathrm{M}$ & I & $\mathrm{U}$ & I & I & $\mathrm{U}$ & $\mathrm{U}$ & $\mathrm{U}$ & MI & MI & $\mathrm{M}$ & $\mathrm{M}$ & $\mathrm{U}$ & VI & VI & 1 & $\mathrm{M}$ \\
\hline $\mathrm{C} 13$ & MU & VI & VI & $\mathrm{M}$ & $\mathrm{U}$ & VU & VI & VI & $\mathrm{M}$ & $\mathrm{U}$ & VU & MI & I & M & $\mathrm{MU}$ & $\mathrm{M}$ & I & I & I & I & $\mathrm{M}$ & VI & VI & $\mathrm{U}$ & $\mathrm{U}$ & $\mathrm{U}$ & VI & I & $\mathrm{U}$ & $\mathrm{U}$ & $\mathrm{U}$ & VI & VI & MU & M \\
\hline C14 & MI & VI & I & I & I & $\mathrm{M}$ & VI & I & I & MI & $\mathrm{U}$ & VI & MI & MI & MI & MI & VI & I & I & MI & I & VI & I & MI & MI & I & VI & I & MI & MI & I & VI & I & MI & MI \\
\hline $\mathrm{C} 15$ & $\mathrm{U}$ & I & I & $\mathrm{U}$ & M & $\mathrm{U}$ & VI & MI & MU & M & VU & I & VI & $\mathrm{U}$ & MU & $\mathrm{M}$ & I & VI & M & M & M & VI & I & $\mathrm{U}$ & $\mathrm{M}$ & $\mathrm{U}$ & I & MI & M & M & $\mathrm{U}$ & I & M & I & $\mathrm{MU}$ \\
\hline
\end{tabular}


Table 3. Cont

\begin{tabular}{|c|c|c|c|c|c|c|c|c|c|c|c|c|c|c|c|c|c|c|c|c|c|c|c|c|c|c|c|c|c|c|c|c|c|c|c|}
\hline & \multicolumn{5}{|c|}{ Expert 1} & \multicolumn{5}{|c|}{ Expert 2} & \multicolumn{5}{|c|}{ Expert 3} & \multicolumn{5}{|c|}{ Expert 4} & \multicolumn{5}{|c|}{ Expert 5} & \multicolumn{5}{|c|}{ Expert 6} & \multicolumn{5}{|c|}{ Expert 7} \\
\hline & AS1 & AS2 & AS3 & AS4 & & AS1 & AS2 & AS3 & AS4 & AS5 & AS1 & AS2 & AS3 & AS4 & & AS1 & AS2 & AS3 & AS4 & AS5 & AS1 & AS2 & AS3 & AS4 & AS5 & AS1 & AS2 & AS3 & AS4 & AS5 & AS1 & AS2 & AS3 & AS4 & AS5 \\
\hline & $\mathrm{U}$ & I & VI & M & MU & $\mathrm{U}$ & I & I & MU & M & VU & I & M & MU & MU & $\mathrm{U}$ & I & VI & M & I & M & VI & I & M & $\mathrm{U}$ & $\mathrm{U}$ & I & MI & M & $\mathrm{U}$ & $\mathrm{U}$ & I & I & I & MU \\
\hline & M & VI & I & M & $\mathrm{M}$ & M & I & I & $\mathrm{M}$ & MU & $M$ & M & M & MU & 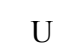 & M & I & $\mathrm{I}$ & M & M & 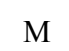 & & $\mathrm{I}$ & $\mathrm{U}$ & M & 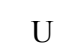 & I & I & $\mathrm{U}$ & $\mathrm{U}$ & 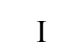 & I & M & M & $\mathrm{M}$ \\
\hline $\mathrm{C} 18$ & MU & I & VI & M & MU & M & I & I & MU & MU & $\mathrm{VU}$ & M & M & $\mathrm{M}$ & MU & $\mathrm{U}$ & I & I & M & VI & $\mathrm{U}$ & VI & 1 & M & U & U & 1 & 1 & $\mathrm{M}$ & $\mathrm{U}$ & 1 & VI & I & I & MU \\
\hline C19 & M & I & VI & MU & $\mathrm{U}$ & VU & VI & VI & $\mathrm{U}$ & MU & $\mathrm{VU}$ & I & VI & MU & $\mathrm{M}$ & M & I & I & I & M & $\mathrm{U}$ & I & I & M & M & $\mathrm{U}$ & MI & I & $\mathrm{U}$ & $\mathrm{U}$ & I & I & M & MU & MU \\
\hline C20 & $\mathrm{M}$ & VI & VI & $\mathrm{M}$ & $\mathrm{M}$ & VU & VI & I & MU & MU & $\mathrm{VU}$ & I & VI & MU & & $\mathrm{U}$ & VI & I & I & $\mathrm{M}$ & $\mathrm{U}$ & & VI & $\mathrm{U}$ & L & $\mathrm{U}$ & I & 1 & $\mathrm{U}$ & $\mathrm{M}$ & $x$ & I & $\mathrm{M}$ & $\mathrm{MU}$ & $\mathrm{M}$ \\
\hline & MU & I & VI & $\mathrm{U}$ & & & VI & & MU & $\mathrm{U}$ & $\mathrm{U}$ & VI & VI & & & $\mathrm{M}$ & $\sqrt{1}$ & & $\mathrm{M}$ & & $\mathrm{U}$ & VI & 1 & $\mathrm{U}$ & $\mathrm{NI}$ & 0 & VI & MI & $\mathrm{U}$ & $\mathrm{U}$ & MU & 1 & VI & 1 & I \\
\hline $\mathrm{C} 22$ & I & M & MI & MU & MU & I & I & $\mathrm{M}$ & $\mathrm{MU}$ & MU & I & I & M & MU & MU & 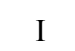 & I & MI & MU & MU & MI & I & MI & MU & MU & I & I & $\mathrm{M}$ & MU & MU & $\mathrm{M}$ & I & $\mathrm{M}$ & $\mathrm{M}$ & I \\
\hline $\mathrm{C} 23$ & $\mathrm{M}$ & VI & I & $\mathrm{M}$ & MU & $\mathrm{VU}$ & VI & I & $\mathrm{MU}$ & $\mathrm{M}$ & $\mathrm{U}$ & VI & I & $\mathrm{U}$ & $\mathrm{M}$ & $\mathrm{M}$ & I & I & $\mathrm{M}$ & $\mathrm{M}$ & $\mathrm{M}$ & VI & I & $\mathrm{M}$ & $\mathrm{U}$ & $\mathrm{U}$ & I & I & $\mathrm{U}$ & $\mathrm{U}$ & I & $\mathrm{M}$ & I & I & $\mathrm{MU}$ \\
\hline & MU & I & MU & MU & MU & M & VI & $\mathrm{M}$ & MU & $\mathrm{U}$ & $\mathrm{U}$ & VI & M & $\mathrm{M}$ & MU & $\mathrm{U}$ & I & M & M & I & $\mathrm{U}$ & I & M & $\mathrm{U}$ & $\mathrm{M}$ & $\mathrm{U}$ & I & I & $\mathrm{M}$ & M & 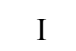 & I & I & I & MU \\
\hline $\mathrm{C} 25$ & MU & I & I & $\mathrm{U}$ & $\mathrm{M}$ & $\mathrm{M}$ & I & VI & $\mathrm{U}$ & $\mathrm{M}$ & VU & I & $\mathrm{M}$ & $\mathrm{M}$ & $\mathrm{M}$ & $\mathrm{M}$ & I & I & M & I & $\mathrm{U}$ & I & I & $\mathrm{M}$ & $\mathrm{U}$ & $\mathrm{U}$ & I & I & $\mathrm{U}$ & $\mathrm{U}$ & 1 & I & $\mathrm{M}$ & I & I \\
\hline $\mathrm{C} 26$ & $\mathrm{U}$ & I & M & M & MU & M & VI & I & $\mathrm{MU}$ & $\mathrm{M}$ & M & I & MI & M & $\mathrm{MU}$ & $\mathrm{M}$ & I & M & I & M & $\mathrm{M}$ & VI & MI & $\mathrm{VU}$ & $\mathrm{U}$ & $\mathrm{U}$ & I & MI & VU & $\mathrm{U}$ & I & I & $\mathrm{M}$ & $\mathrm{M}$ & $\mathrm{M}$ \\
\hline $\mathrm{C} 27$ & MU & I & I & $\mathrm{MU}$ & MU & M & I & MI & MU & MU & $\mathrm{M}$ & $\mathrm{M}$ & VI & $\mathrm{MU}$ & $\mathrm{U}$ & $\mathrm{M}$ & I & I & M & I & $\mathrm{M}$ & VI & VI & $\mathrm{U}$ & $\mathrm{U}$ & $\mathrm{U}$ & VI & I & $\mathrm{MU}$ & $\mathrm{M}$ & $\mathrm{U}$ & I & I & $\mathrm{M}$ & $\mathrm{MU}$ \\
\hline $\mathrm{C} 28$ & $\mathrm{U}$ & I & VI & $\mathrm{M}$ & $\mathrm{U}$ & $\mathrm{U}$ & VI & I & $\mathrm{U}$ & MU & $\mathrm{M}$ & $\mathrm{M}$ & $\mathrm{M}$ & $\mathrm{M}$ & $\mathrm{M}$ & $\mathrm{M}$ & VI & VI & 1 & I & $\mathrm{M}$ & VI & VI & $\mathrm{M}$ & $\mathrm{U}$ & $\mathrm{U}$ & MI & MI & $\mathrm{U}$ & MU & $\mathrm{U}$ & I & I & $\mathrm{MU}$ & I \\
\hline C29 & $\mathrm{U}$ & VI & I & $\mathrm{U}$ & MU & $\mathrm{U}$ & VI & I & $\mathrm{U}$ & $\mathrm{M}$ & VU & I & $\mathrm{M}$ & $\mathrm{U}$ & MU & $\mathrm{M}$ & I & I & $\mathrm{M}$ & M & $\mathrm{M}$ & VI & VI & $\mathrm{M}$ & $\mathrm{M}$ & $\mathrm{U}$ & MI & MI & M & $\mathrm{U}$ & I & I & I & MU & $\mathrm{MU}$ \\
\hline C30 & $\mathrm{M}$ & I & VI & M & $\mathrm{U}$ & VU & VI & MI & $\mathrm{U}$ & MU & $\mathrm{U}$ & M & M & M & $\mathrm{U}$ & M & VI & I & M & M & $\mathrm{M}$ & I & VI & $\mathrm{U}$ & $\mathrm{M}$ & $\mathrm{U}$ & I & I & $\mathrm{M}$ & MU & MU & $\mathrm{M}$ & I & M & I \\
\hline
\end{tabular}


Table 4. Converting interval-valued triangular fuzzy numbers from expert responses.

\begin{tabular}{|c|c|c|c|c|c|c|c|c|c|c|c|c|c|c|c|}
\hline & \multicolumn{5}{|c|}{ AS1 } & \multicolumn{5}{|c|}{ AS2 } & \multicolumn{5}{|c|}{ AS3 } \\
\hline & $x_{i j}$ & $\tilde{\boldsymbol{x}}_{i j}$ & $y_{i j}$ & $\tilde{\mathbf{z}}_{i j}$ & $z_{i j}$ & $x_{i j}$ & $\tilde{x}_{i j}$ & $y_{i j}$ & $\tilde{\mathbf{z}}_{i j}$ & $z_{i j}$ & $x_{i j}$ & $\tilde{x}_{i j}$ & $y_{i j}$ & $\tilde{\mathbf{z}}_{i j}$ & $z_{i j}$ \\
\hline $\mathrm{C} 1$ & 0.2214 & 0.3143 & 0.4286 & 0.5571 & 0.6429 & 0.7214 & 0.8643 & 0.9571 & 0.9786 & 1.0000 & 0.5357 & 0.6643 & 0.8000 & 0.8714 & 0.9714 \\
\hline $\mathrm{C} 2$ & 0.1786 & 0.2714 & 0.4000 & 0.5429 & 0.6357 & 0.6357 & 0.7786 & 0.8857 & 0.9286 & 0.9643 & 0.5500 & 0.6929 & 0.8286 & 0.8929 & 0.9786 \\
\hline $\mathrm{C} 3$ & 0.0714 & 0.1357 & 0.2143 & 0.3643 & 0.4643 & 0.7214 & 0.8643 & 0.9571 & 0.9786 & 1.0000 & 0.7071 & 0.8357 & 0.9286 & 0.9571 & 0.9929 \\
\hline $\mathrm{C} 4$ & 0.2214 & 0.3214 & 0.4429 & 0.5786 & 0.6714 & 0.4929 & 0.6643 & 0.8143 & 0.8857 & 0.9571 & 0.3357 & 0.4643 & 0.6143 & 0.7357 & 0.8214 \\
\hline C5 & 0.0714 & 0.1357 & 0.2143 & 0.3643 & 0.4643 & 0.6643 & 0.8071 & 0.9143 & 0.9500 & 0.9929 & 0.7500 & 0.8643 & 0.9429 & 0.9643 & 0.9929 \\
\hline C6 & 0.0357 & 0.0929 & 0.1571 & 0.2929 & 0.3786 & 0.7643 & 0.8929 & 0.9714 & 0.9857 & 1.0000 & 0.7071 & 0.8357 & 0.9286 & 0.9571 & 0.9929 \\
\hline $\mathrm{C} 7$ & 0.1500 & 0.2286 & 0.3143 & 0.4429 & 0.5286 & 0.5357 & 0.6929 & 0.8286 & 0.8929 & 0.9571 & 0.5929 & 0.7786 & 0.9143 & 0.9571 & 1.0000 \\
\hline $\mathrm{C} 8$ & 0.0714 & 0.1286 & 0.2000 & 0.3429 & 0.4357 & 0.6643 & 0.8071 & 0.9143 & 0.9500 & 0.9929 & 0.6643 & 0.8071 & 0.9143 & 0.9500 & 0.9929 \\
\hline C9 & 0.5500 & 0.7500 & 0.9000 & 0.9500 & 1.0000 & 0.8500 & 0.9500 & 1.0000 & 1.0000 & 1.0000 & 0.8500 & 0.9500 & 1.0000 & 1.0000 & 1.0000 \\
\hline $\mathrm{C} 10$ & 0.1857 & 0.2857 & 0.4000 & 0.5286 & 0.6143 & 0.6357 & 0.8071 & 0.9286 & 0.9643 & 1.0000 & 0.4929 & 0.6643 & 0.8143 & 0.8857 & 0.9571 \\
\hline $\mathrm{C} 11$ & 0.0357 & 0.0786 & 0.1286 & 0.2643 & 0.3500 & 0.7071 & 0.8357 & 0.9286 & 0.9571 & 0.9929 & 0.6500 & 0.7786 & 0.8857 & 0.9286 & 0.9857 \\
\hline $\mathrm{C} 12$ & 0.1071 & 0.1714 & 0.2571 & 0.4000 & 0.4929 & 0.6643 & 0.8071 & 0.9143 & 0.9500 & 0.9929 & 0.6643 & 0.8071 & 0.9143 & 0.9500 & 0.9929 \\
\hline $\mathrm{C} 13$ & 0.0714 & 0.1357 & 0.2143 & 0.3500 & 0.4357 & 0.7500 & 0.8643 & 0.9429 & 0.9643 & 0.9929 & 0.7214 & 0.8643 & 0.9571 & 0.9786 & 1.0000 \\
\hline C14 & 0.4000 & 0.5357 & 0.6714 & 0.7643 & 0.8571 & 0.8500 & 0.9500 & 1.0000 & 1.0000 & 1.0000 & 0.5357 & 0.7214 & 0.8714 & 0.9286 & 0.9929 \\
\hline $\mathrm{C} 15$ & 0.0714 & 0.1286 & 0.2000 & 0.3429 & 0.4357 & 0.6357 & 0.8071 & 0.9286 & 0.9643 & 1.0000 & 0.5643 & 0.6929 & 0.8143 & 0.8786 & 0.9500 \\
\hline $\mathrm{C} 16$ & 0.0357 & 0.0857 & 0.1429 & 0.2857 & 0.3786 & 0.5929 & 0.7786 & 0.9143 & 0.9571 & 1.0000 & 0.5786 & 0.7214 & 0.8429 & 0.9000 & 0.9571 \\
\hline $\mathrm{C} 17$ & 0.2571 & 0.3643 & 0.5000 & 0.6357 & 0.7286 & 0.5500 & 0.7214 & 0.8571 & 0.9143 & 0.9643 & 0.4643 & 0.6357 & 0.7857 & 0.8643 & 0.9286 \\
\hline $\mathrm{C} 18$ & 0.1143 & 0.2000 & 0.2857 & 0.4143 & 0.5000 & 0.5929 & 0.7500 & 0.8714 & 0.9214 & 0.9643 & 0.5500 & 0.7214 & 0.8571 & 0.9143 & 0.9643 \\
\hline C19 & 0.1500 & 0.2214 & 0.3000 & 0.4214 & 0.5000 & 0.5786 & 0.7500 & 0.8857 & 0.9357 & 0.9929 & 0.6357 & 0.7786 & 0.8857 & 0.9286 & 0.9643 \\
\hline C20 & 0.1143 & 0.1786 & 0.2429 & 0.3643 & 0.4429 & 0.6786 & 0.8357 & 0.9429 & 0.9714 & 1.0000 & 0.6357 & 0.7786 & 0.8857 & 0.9286 & 0.9643 \\
\hline $\mathrm{C} 21$ & 0.0357 & 0.1143 & 0.2000 & 0.3429 & 0.4357 & 0.7643 & 0.8929 & 0.9714 & 0.9857 & 1.0000 & 0.6929 & 0.8071 & 0.9000 & 0.9357 & 0.9857 \\
\hline $\mathrm{C} 22$ & 0.4929 & 0.6643 & 0.8143 & 0.8857 & 0.9571 & 0.5071 & 0.6929 & 0.8429 & 0.9071 & 0.9643 & 0.3357 & 0.4357 & 0.5857 & 0.7143 & 0.8357 \\
\hline $\mathrm{C} 23$ & 0.1857 & 0.2714 & 0.3714 & 0.5000 & 0.5857 & 0.6786 & 0.8071 & 0.9000 & 0.9357 & 0.9643 & 0.5500 & 0.7500 & 0.9000 & 0.9500 & 1.0000 \\
\hline $\mathrm{C} 24$ & 0.1143 & 0.2071 & 0.3000 & 0.4357 & 0.5286 & 0.6357 & 0.8071 & 0.9286 & 0.9643 & 1.0000 & 0.3000 & 0.4357 & 0.5857 & 0.7071 & 0.7929 \\
\hline $\mathrm{C} 25$ & 0.1500 & 0.2429 & 0.3429 & 0.4714 & 0.5571 & 0.5500 & 0.7500 & 0.9000 & 0.9500 & 1.0000 & 0.5071 & 0.6643 & 0.8000 & 0.8714 & 0.9286 \\
\hline C26 & 0.2214 & 0.3214 & 0.4429 & 0.5786 & 0.6714 & 0.6357 & 0.8071 & 0.9286 & 0.9643 & 1.0000 & 0.3786 & 0.4929 & 0.6429 & 0.7571 & 0.8714 \\
\hline $\mathrm{C} 27$ & 0.1429 & 0.2357 & 0.3571 & 0.5071 & 0.6071 & 0.5929 & 0.7500 & 0.8714 & 0.9214 & 0.9643 & 0.6214 & 0.7786 & 0.9000 & 0.9429 & 0.9929 \\
\hline $\mathrm{C} 28$ & 0.1071 & 0.1786 & 0.2714 & 0.4214 & 0.5214 & 0.6214 & 0.7500 & 0.8571 & 0.9071 & 0.9571 & 0.6214 & 0.7500 & 0.8571 & 0.9071 & 0.9571 \\
\hline C29 & 0.1500 & 0.2286 & 0.3143 & 0.4429 & 0.5286 & 0.6643 & 0.8071 & 0.9143 & 0.9500 & 0.9929 & 0.5357 & 0.6929 & 0.8286 & 0.8929 & 0.9571 \\
\hline $\mathrm{C} 30$ & 0.1071 & 0.1857 & 0.2857 & 0.4286 & 0.5214 & 0.5500 & 0.6929 & 0.8143 & 0.8786 & 0.9286 & 0.5786 & 0.7214 & 0.8429 & 0.9000 & 0.9571 \\
\hline
\end{tabular}


Table 4. Cont.

\begin{tabular}{|c|c|c|c|c|c|c|c|c|c|c|}
\hline & \multicolumn{5}{|c|}{ AS4 } & \multicolumn{5}{|c|}{ AS5 } \\
\hline & $x_{i j}$ & $\tilde{\boldsymbol{x}}_{i j}$ & $y_{i j}$ & $\tilde{z}_{i j}$ & $z_{i j}$ & $x_{i j}$ & $\tilde{x}_{i j}$ & $y_{i j}$ & $\tilde{z}_{i j}$ & $z_{i j}$ \\
\hline $\mathrm{C} 1$ & 0.1071 & 0.1929 & 0.3000 & 0.4500 & 0.5500 & 0.1429 & 0.2357 & 0.3571 & 0.5071 & 0.6071 \\
\hline $\mathrm{C} 2$ & 0.1500 & 0.2429 & 0.3429 & 0.4714 & 0.5571 & 0.0714 & 0.1286 & 0.2000 & 0.3429 & 0.4357 \\
\hline $\mathrm{C} 3$ & 0.1071 & 0.2071 & 0.3286 & 0.4786 & 0.5786 & 0.1786 & 0.2786 & 0.4143 & 0.5643 & 0.6643 \\
\hline $\mathrm{C} 4$ & 0.1071 & 0.1714 & 0.2571 & 0.3857 & 0.4643 & 0.0357 & 0.0857 & 0.1429 & 0.2714 & 0.3500 \\
\hline $\mathrm{C} 5$ & 0.1500 & 0.2643 & 0.3857 & 0.5214 & 0.6143 & 0.1500 & 0.2643 & 0.3857 & 0.5214 & 0.6143 \\
\hline C6 & 0.2286 & 0.3643 & 0.5000 & 0.6214 & 0.7071 & 0.1071 & 0.2071 & 0.3286 & 0.4786 & 0.5786 \\
\hline $\mathrm{C} 7$ & 0.1929 & 0.3071 & 0.4286 & 0.5571 & 0.6429 & 0.1071 & 0.2071 & 0.3286 & 0.4786 & 0.5786 \\
\hline $\mathrm{C} 8$ & 0.3429 & 0.4929 & 0.6429 & 0.7500 & 0.8286 & 0.1857 & 0.2929 & 0.4143 & 0.5500 & 0.6429 \\
\hline C9 & 0.5357 & 0.7214 & 0.8714 & 0.9286 & 0.9929 & 0.5500 & 0.7500 & 0.9000 & 0.9500 & 1.0000 \\
\hline $\mathrm{C} 10$ & 0.1500 & 0.2429 & 0.3429 & 0.4714 & 0.5571 & 0.0714 & 0.1429 & 0.2286 & 0.3714 & 0.4643 \\
\hline C11 & 0.1071 & 0.2071 & 0.3286 & 0.4786 & 0.5786 & 0.1857 & 0.2786 & 0.3857 & 0.5214 & 0.6143 \\
\hline $\mathrm{C} 12$ & 0.1500 & 0.2643 & 0.3857 & 0.5214 & 0.6143 & 0.2214 & 0.3357 & 0.4714 & 0.6071 & 0.7000 \\
\hline $\mathrm{C} 13$ & 0.1857 & 0.2929 & 0.4143 & 0.5500 & 0.6429 & 0.1143 & 0.2071 & 0.3000 & 0.4357 & 0.5286 \\
\hline C14 & 0.4929 & 0.6357 & 0.7857 & 0.8643 & 0.9714 & 0.4643 & 0.5786 & 0.7286 & 0.8214 & 0.9571 \\
\hline $\mathrm{C} 15$ & 0.1500 & 0.2500 & 0.3571 & 0.4929 & 0.5857 & 0.1786 & 0.2929 & 0.4429 & 0.5929 & 0.6929 \\
\hline C16 & 0.2214 & 0.3500 & 0.5000 & 0.6357 & 0.7286 & 0.1143 & 0.2357 & 0.3571 & 0.4929 & 0.5857 \\
\hline $\mathrm{C} 17$ & 0.1429 & 0.2357 & 0.3571 & 0.5071 & 0.6071 & 0.1429 & 0.2357 & 0.3571 & 0.5071 & 0.6071 \\
\hline C18 & 0.2571 & 0.3786 & 0.5286 & 0.6643 & 0.7571 & 0.1214 & 0.2357 & 0.3429 & 0.4714 & 0.5571 \\
\hline C19 & 0.1143 & 0.2357 & 0.3571 & 0.4929 & 0.5857 & 0.1071 & 0.2071 & 0.3286 & 0.4786 & 0.5786 \\
\hline $\mathrm{C} 20$ & 0.1143 & 0.2357 & 0.3571 & 0.4929 & 0.5857 & 0.1429 & 0.2500 & 0.3857 & 0.5357 & 0.6357 \\
\hline $\mathrm{C} 21$ & 0.1143 & 0.2214 & 0.3286 & 0.4643 & 0.5571 & 0.1500 & 0.2643 & 0.3857 & 0.5214 & 0.6143 \\
\hline $\mathrm{C} 22$ & 0.0357 & 0.1786 & 0.3286 & 0.4786 & 0.5786 & 0.0786 & 0.2357 & 0.3857 & 0.5214 & 0.6143 \\
\hline $\mathrm{C} 23$ & 0.1857 & 0.2929 & 0.4143 & 0.5500 & 0.6429 & 0.1071 & 0.2071 & 0.3286 & 0.4786 & 0.5786 \\
\hline $\mathrm{C} 24$ & 0.1857 & 0.3071 & 0.4429 & 0.5786 & 0.6714 & 0.1500 & 0.2786 & 0.4143 & 0.5500 & 0.6429 \\
\hline $\mathrm{C} 25$ & 0.1857 & 0.2786 & 0.3857 & 0.5214 & 0.6143 & 0.2643 & 0.3786 & 0.5000 & 0.6214 & 0.7071 \\
\hline $\mathrm{C} 26$ & 0.1857 & 0.2786 & 0.3857 & 0.5071 & 0.5857 & 0.1071 & 0.2071 & 0.3286 & 0.4786 & 0.5786 \\
\hline $\mathrm{C} 27$ & 0.0714 & 0.1929 & 0.3286 & 0.4786 & 0.5786 & 0.1143 & 0.2357 & 0.3571 & 0.4929 & 0.5857 \\
\hline $\mathrm{C} 28$ & 0.1857 & 0.2929 & 0.4143 & 0.5500 & 0.6429 & 0.1929 & 0.3214 & 0.4429 & 0.5643 & 0.6500 \\
\hline $\mathrm{C} 29$ & 0.1071 & 0.1929 & 0.3000 & 0.4500 & 0.5500 & 0.1071 & 0.2214 & 0.3571 & 0.5071 & 0.6071 \\
\hline $\mathrm{C} 30$ & 0.1786 & 0.2643 & 0.3857 & 0.5357 & 0.6357 & 0.1500 & 0.2643 & 0.3857 & 0.5214 & 0.6143 \\
\hline
\end{tabular}


Table 5. Benefit matrix.

\begin{tabular}{|c|c|c|c|c|c|c|c|c|c|c|c|c|c|c|c|}
\hline & \multicolumn{5}{|c|}{ AS1 } & \multicolumn{5}{|c|}{ AS2 } & \multicolumn{5}{|c|}{ AS3 } \\
\hline & $x_{i j}$ & $\tilde{x}_{i j}$ & $y_{i j}$ & $\tilde{\boldsymbol{z}}_{i j}$ & $z_{i j}$ & $x_{i j}$ & $\tilde{\boldsymbol{x}}_{i j}$ & $y_{i j}$ & $\tilde{z}_{i j}$ & $z_{i j}$ & $x_{i j}$ & $\tilde{x}_{i j}$ & $y_{i j}$ & $\tilde{z}_{i j}$ & $z_{i j}$ \\
\hline $\mathrm{C} 1$ & 0.2214 & 0.3143 & 0.4286 & 0.5571 & 0.6429 & 0.7214 & 0.8643 & 0.9571 & 0.9786 & 1.0000 & 0.5357 & 0.6643 & 0.8000 & 0.8714 & 0.9714 \\
\hline $\mathrm{C} 2$ & 0.1786 & 0.2714 & 0.4000 & 0.5429 & 0.6357 & 0.6357 & 0.7786 & 0.8857 & 0.9286 & 0.9643 & 0.5500 & 0.6929 & 0.8286 & 0.8929 & 0.9786 \\
\hline $\mathrm{C} 3$ & 0.0714 & 0.1357 & 0.2143 & 0.3643 & 0.4643 & 0.7214 & 0.8643 & 0.9571 & 0.9786 & 1.0000 & 0.7071 & 0.8357 & 0.9286 & 0.9571 & 0.9929 \\
\hline $\mathrm{C} 4$ & 0.2214 & 0.3214 & 0.4429 & 0.5786 & 0.6714 & 0.4929 & 0.6643 & 0.8143 & 0.8857 & 0.9571 & 0.3357 & 0.4643 & 0.6143 & 0.7357 & 0.8214 \\
\hline C5 & 0.0714 & 0.1357 & 0.2143 & 0.3643 & 0.4643 & 0.6643 & 0.8071 & 0.9143 & 0.9500 & 0.9929 & 0.7500 & 0.8643 & 0.9429 & 0.9643 & 0.9929 \\
\hline C6 & 0.0357 & 0.0929 & 0.1571 & 0.2929 & 0.3786 & 0.7643 & 0.8929 & 0.9714 & 0.9857 & 1.0000 & 0.7071 & 0.8357 & 0.9286 & 0.9571 & 0.9929 \\
\hline $\mathrm{C} 7$ & 0.1500 & 0.2286 & 0.3143 & 0.4429 & 0.5286 & 0.5357 & 0.6929 & 0.8286 & 0.8929 & 0.9571 & 0.5929 & 0.7786 & 0.9143 & 0.9571 & 1.0000 \\
\hline $\mathrm{C} 8$ & 0.0714 & 0.1286 & 0.2000 & 0.3429 & 0.4357 & 0.6643 & 0.8071 & 0.9143 & 0.9500 & 0.9929 & 0.6643 & 0.8071 & 0.9143 & 0.9500 & 0.9929 \\
\hline C9 & 0.5500 & 0.7500 & 0.9000 & 0.9500 & 1.0000 & 0.8500 & 0.9500 & 1.0000 & 1.0000 & 1.0000 & 0.8500 & 0.9500 & 1.0000 & 1.0000 & 1.0000 \\
\hline $\mathrm{C} 10$ & 0.1857 & 0.2857 & 0.4000 & 0.5286 & 0.6143 & 0.6357 & 0.8071 & 0.9286 & 0.9643 & 1.0000 & 0.4929 & 0.6643 & 0.8143 & 0.8857 & 0.9571 \\
\hline C11 & 0.0357 & 0.0786 & 0.1286 & 0.2643 & 0.3500 & 0.7071 & 0.8357 & 0.9286 & 0.9571 & 0.9929 & 0.6500 & 0.7786 & 0.8857 & 0.9286 & 0.9857 \\
\hline $\mathrm{C} 12$ & 0.1071 & 0.1714 & 0.2571 & 0.4000 & 0.4929 & 0.6643 & 0.8071 & 0.9143 & 0.9500 & 0.9929 & 0.6643 & 0.8071 & 0.9143 & 0.9500 & 0.9929 \\
\hline $\mathrm{C} 13$ & 0.0714 & 0.1357 & 0.2143 & 0.3500 & 0.4357 & 0.7500 & 0.8643 & 0.9429 & 0.9643 & 0.9929 & 0.7214 & 0.8643 & 0.9571 & 0.9786 & 1.0000 \\
\hline $\mathrm{C} 14$ & 0.4000 & 0.5357 & 0.6714 & 0.7643 & 0.8571 & 0.8500 & 0.9500 & 1.0000 & 1.0000 & 1.0000 & 0.5357 & 0.7214 & 0.8714 & 0.9286 & 0.9929 \\
\hline $\mathrm{C} 15$ & 0.0714 & 0.1286 & 0.2000 & 0.3429 & 0.4357 & 0.6357 & 0.8071 & 0.9286 & 0.9643 & 1.0000 & 0.5643 & 0.6929 & 0.8143 & 0.8786 & 0.9500 \\
\hline $\mathrm{C} 16$ & 0.0357 & 0.0857 & 0.1429 & 0.2857 & 0.3786 & 0.5929 & 0.7786 & 0.9143 & 0.9571 & 1.0000 & 0.5786 & 0.7214 & 0.8429 & 0.9000 & 0.9571 \\
\hline $\mathrm{C} 17$ & 0.2571 & 0.3643 & 0.5000 & 0.6357 & 0.7286 & 0.5500 & 0.7214 & 0.8571 & 0.9143 & 0.9643 & 0.4643 & 0.6357 & 0.7857 & 0.8643 & 0.9286 \\
\hline $\mathrm{C} 18$ & 0.1143 & 0.2000 & 0.2857 & 0.4143 & 0.5000 & 0.5929 & 0.7500 & 0.8714 & 0.9214 & 0.9643 & 0.5500 & 0.7214 & 0.8571 & 0.9143 & 0.9643 \\
\hline C19 & 0.1500 & 0.2214 & 0.3000 & 0.4214 & 0.5000 & 0.5786 & 0.7500 & 0.8857 & 0.9357 & 0.9929 & 0.6357 & 0.7786 & 0.8857 & 0.9286 & 0.9643 \\
\hline $\mathrm{C} 20$ & 0.1143 & 0.1786 & 0.2429 & 0.3643 & 0.4429 & 0.6786 & 0.8357 & 0.9429 & 0.9714 & 1.0000 & 0.6357 & 0.7786 & 0.8857 & 0.9286 & 0.9643 \\
\hline $\mathrm{C} 21$ & 0.0357 & 0.1143 & 0.2000 & 0.3429 & 0.4357 & 0.7643 & 0.8929 & 0.9714 & 0.9857 & 1.0000 & 0.6929 & 0.8071 & 0.9000 & 0.9357 & 0.9857 \\
\hline $\mathrm{C} 22$ & 0.4929 & 0.6643 & 0.8143 & 0.8857 & 0.9571 & 0.5071 & 0.6929 & 0.8429 & 0.9071 & 0.9643 & 0.3357 & 0.4357 & 0.5857 & 0.7143 & 0.8357 \\
\hline $\mathrm{C} 23$ & 0.1857 & 0.2714 & 0.3714 & 0.5000 & 0.5857 & 0.6786 & 0.8071 & 0.9000 & 0.9357 & 0.9643 & 0.5500 & 0.7500 & 0.9000 & 0.9500 & 1.0000 \\
\hline $\mathrm{C} 24$ & 0.1143 & 0.2071 & 0.3000 & 0.4357 & 0.5286 & 0.6357 & 0.8071 & 0.9286 & 0.9643 & 1.0000 & 0.3000 & 0.4357 & 0.5857 & 0.7071 & 0.7929 \\
\hline $\mathrm{C} 25$ & 0.1500 & 0.2429 & 0.3429 & 0.4714 & 0.5571 & 0.5500 & 0.7500 & 0.9000 & 0.9500 & 1.0000 & 0.5071 & 0.6643 & 0.8000 & 0.8714 & 0.9286 \\
\hline $\mathrm{C} 26$ & 0.2214 & 0.3214 & 0.4429 & 0.5786 & 0.6714 & 0.6357 & 0.8071 & 0.9286 & 0.9643 & 1.0000 & 0.3786 & 0.4929 & 0.6429 & 0.7571 & 0.8714 \\
\hline $\mathrm{C} 27$ & 0.1429 & 0.2357 & 0.3571 & 0.5071 & 0.6071 & 0.5929 & 0.7500 & 0.8714 & 0.9214 & 0.9643 & 0.6214 & 0.7786 & 0.9000 & 0.9429 & 0.9929 \\
\hline $\mathrm{C} 28$ & 0.1071 & 0.1786 & 0.2714 & 0.4214 & 0.5214 & 0.6214 & 0.7500 & 0.8571 & 0.9071 & 0.9571 & 0.6214 & 0.7500 & 0.8571 & 0.9071 & 0.9571 \\
\hline C29 & 0.1500 & 0.2286 & 0.3143 & 0.4429 & 0.5286 & 0.6643 & 0.8071 & 0.9143 & 0.9500 & 0.9929 & 0.5357 & 0.6929 & 0.8286 & 0.8929 & 0.9571 \\
\hline $\mathrm{C} 30$ & 0.1071 & 0.1857 & 0.2857 & 0.4286 & 0.5214 & 0.5500 & 0.6929 & 0.8143 & 0.8786 & 0.9286 & 0.5786 & 0.7214 & 0.8429 & 0.9000 & 0.9571 \\
\hline
\end{tabular}


Table 5. Cont.

\begin{tabular}{|c|c|c|c|c|c|c|c|c|c|c|}
\hline & \multicolumn{5}{|c|}{ AS4 } & \multicolumn{5}{|c|}{ AS5 } \\
\hline & $x_{i j}$ & $\tilde{x}_{i j}$ & $y_{i j}$ & $\tilde{z}_{i j}$ & $z_{i j}$ & $x_{i j}$ & $\tilde{x}_{i j}$ & $y_{i j}$ & $\tilde{\boldsymbol{z}}_{i j}$ & $z_{i j}$ \\
\hline $\mathrm{C} 1$ & 0.1071 & 0.1929 & 0.3000 & 0.4500 & 0.5500 & 0.1429 & 0.2357 & 0.3571 & 0.5071 & 0.6071 \\
\hline $\mathrm{C} 2$ & 0.1500 & 0.2429 & 0.3429 & 0.4714 & 0.5571 & 0.0714 & 0.1286 & 0.2000 & 0.3429 & 0.4357 \\
\hline $\mathrm{C} 3$ & 0.1071 & 0.2071 & 0.3286 & 0.4786 & 0.5786 & 0.1786 & 0.2786 & 0.4143 & 0.5643 & 0.6643 \\
\hline $\mathrm{C} 4$ & 0.1071 & 0.1714 & 0.2571 & 0.3857 & 0.4643 & 0.0357 & 0.0857 & 0.1429 & 0.2714 & 0.3500 \\
\hline C5 & 0.1500 & 0.2643 & 0.3857 & 0.5214 & 0.6143 & 0.1500 & 0.2643 & 0.3857 & 0.5214 & 0.6143 \\
\hline C6 & 0.2286 & 0.3643 & 0.5000 & 0.6214 & 0.7071 & 0.1071 & 0.2071 & 0.3286 & 0.4786 & 0.5786 \\
\hline $\mathrm{C} 7$ & 0.1929 & 0.3071 & 0.4286 & 0.5571 & 0.6429 & 0.1071 & 0.2071 & 0.3286 & 0.4786 & 0.5786 \\
\hline $\mathrm{C} 8$ & 0.3429 & 0.4929 & 0.6429 & 0.7500 & 0.8286 & 0.1857 & 0.2929 & 0.4143 & 0.5500 & 0.6429 \\
\hline C9 & 0.5357 & 0.7214 & 0.8714 & 0.9286 & 0.9929 & 0.5500 & 0.7500 & 0.9000 & 0.9500 & 1.0000 \\
\hline $\mathrm{C} 10$ & 0.1500 & 0.2429 & 0.3429 & 0.4714 & 0.5571 & 0.0714 & 0.1429 & 0.2286 & 0.3714 & 0.4643 \\
\hline $\mathrm{C} 11$ & 0.1071 & 0.2071 & 0.3286 & 0.4786 & 0.5786 & 0.1857 & 0.2786 & 0.3857 & 0.5214 & 0.6143 \\
\hline $\mathrm{C} 12$ & 0.1500 & 0.2643 & 0.3857 & 0.5214 & 0.6143 & 0.2214 & 0.3357 & 0.4714 & 0.6071 & 0.7000 \\
\hline $\mathrm{C} 13$ & 0.1857 & 0.2929 & 0.4143 & 0.5500 & 0.6429 & 0.1143 & 0.2071 & 0.3000 & 0.4357 & 0.5286 \\
\hline $\mathrm{C} 14$ & 0.4929 & 0.6357 & 0.7857 & 0.8643 & 0.9714 & 0.4643 & 0.5786 & 0.7286 & 0.8214 & 0.9571 \\
\hline $\mathrm{C} 15$ & 0.1500 & 0.2500 & 0.3571 & 0.4929 & 0.5857 & 0.1786 & 0.2929 & 0.4429 & 0.5929 & 0.6929 \\
\hline $\mathrm{C} 16$ & 0.2214 & 0.3500 & 0.5000 & 0.6357 & 0.7286 & 0.1143 & 0.2357 & 0.3571 & 0.4929 & 0.5857 \\
\hline $\mathrm{C} 17$ & 0.1429 & 0.2357 & 0.3571 & 0.5071 & 0.6071 & 0.1429 & 0.2357 & 0.3571 & 0.5071 & 0.6071 \\
\hline $\mathrm{C} 18$ & 0.2571 & 0.3786 & 0.5286 & 0.6643 & 0.7571 & 0.1214 & 0.2357 & 0.3429 & 0.4714 & 0.5571 \\
\hline C19 & 0.1143 & 0.2357 & 0.3571 & 0.4929 & 0.5857 & 0.1071 & 0.2071 & 0.3286 & 0.4786 & 0.5786 \\
\hline $\mathrm{C} 20$ & 0.1143 & 0.2357 & 0.3571 & 0.4929 & 0.5857 & 0.1429 & 0.2500 & 0.3857 & 0.5357 & 0.6357 \\
\hline $\mathrm{C} 21$ & 0.1143 & 0.2214 & 0.3286 & 0.4643 & 0.5571 & 0.1500 & 0.2643 & 0.3857 & 0.5214 & 0.6143 \\
\hline $\mathrm{C} 22$ & 0.0357 & 0.1786 & 0.3286 & 0.4786 & 0.5786 & 0.0786 & 0.2357 & 0.3857 & 0.5214 & 0.6143 \\
\hline $\mathrm{C} 23$ & 0.1857 & 0.2929 & 0.4143 & 0.5500 & 0.6429 & 0.1071 & 0.2071 & 0.3286 & 0.4786 & 0.5786 \\
\hline $\mathrm{C} 24$ & 0.1857 & 0.3071 & 0.4429 & 0.5786 & 0.6714 & 0.1500 & 0.2786 & 0.4143 & 0.5500 & 0.6429 \\
\hline $\mathrm{C} 25$ & 0.1857 & 0.2786 & 0.3857 & 0.5214 & 0.6143 & 0.2643 & 0.3786 & 0.5000 & 0.6214 & 0.7071 \\
\hline $\mathrm{C} 26$ & 0.1857 & 0.2786 & 0.3857 & 0.5071 & 0.5857 & 0.1071 & 0.2071 & 0.3286 & 0.4786 & 0.5786 \\
\hline $\mathrm{C} 27$ & 0.0714 & 0.1929 & 0.3286 & 0.4786 & 0.5786 & 0.1143 & 0.2357 & 0.3571 & 0.4929 & 0.5857 \\
\hline $\mathrm{C} 28$ & 0.1857 & 0.2929 & 0.4143 & 0.5500 & 0.6429 & 0.1929 & 0.3214 & 0.4429 & 0.5643 & 0.6500 \\
\hline C29 & 0.1071 & 0.1929 & 0.3000 & 0.4500 & 0.5500 & 0.1071 & 0.2214 & 0.3571 & 0.5071 & 0.6071 \\
\hline $\mathrm{C} 30$ & 0.1786 & 0.2643 & 0.3857 & 0.5357 & 0.6357 & 0.1500 & 0.2643 & 0.3857 & 0.5214 & 0.6143 \\
\hline
\end{tabular}


Table 6. Cost matrix.

\begin{tabular}{|c|c|c|c|c|c|c|c|c|c|c|c|c|c|c|c|}
\hline & \multicolumn{5}{|c|}{ AS1 } & \multicolumn{5}{|c|}{ AS2 } & \multicolumn{5}{|c|}{ AS3 } \\
\hline & $x_{i j}$ & $\tilde{\boldsymbol{x}}_{i j}$ & $y_{i j}$ & $\tilde{\boldsymbol{z}}_{i j}$ & $z_{i j}$ & $x_{i j}$ & $\tilde{\boldsymbol{x}}_{i j}$ & $y_{i j}$ & $\tilde{z}_{i j}$ & $z_{i j}$ & $x_{i j}$ & $\tilde{\boldsymbol{x}}_{i j}$ & $y_{i j}$ & $\tilde{\boldsymbol{z}}_{i j}$ & $z_{i j}$ \\
\hline $\mathrm{C} 1$ & 0.1613 & 0.1136 & 0.0833 & 0.0641 & 0.0556 & 0.0495 & 0.0413 & 0.0373 & 0.0365 & 0.0357 & 0.0667 & 0.0538 & 0.0446 & 0.0410 & 0.0368 \\
\hline $\mathrm{C} 2$ & 0.2000 & 0.1316 & 0.0893 & 0.0658 & 0.0562 & 0.0562 & 0.0459 & 0.0403 & 0.0385 & 0.0370 & 0.0649 & 0.0515 & 0.0431 & 0.0400 & 0.0365 \\
\hline $\mathrm{C} 3$ & 0.5000 & 0.2632 & 0.1667 & 0.0980 & 0.0769 & 0.0495 & 0.0413 & 0.0373 & 0.0365 & 0.0357 & 0.0505 & 0.0427 & 0.0385 & 0.0373 & 0.0360 \\
\hline $\mathrm{C} 4$ & 0.1613 & 0.1111 & 0.0806 & 0.0617 & 0.0532 & 0.0725 & 0.0538 & 0.0439 & 0.0403 & 0.0373 & 0.1064 & 0.0769 & 0.0581 & 0.0485 & 0.0435 \\
\hline $\mathrm{C} 5$ & 0.5000 & 0.2632 & 0.1667 & 0.0980 & 0.0769 & 0.0538 & 0.0442 & 0.0391 & 0.0376 & 0.0360 & 0.0476 & 0.0413 & 0.0379 & 0.0370 & 0.0360 \\
\hline C6 & 1.0000 & 0.3846 & 0.2273 & 0.1220 & 0.0943 & 0.0467 & 0.0400 & 0.0368 & 0.0362 & 0.0357 & 0.0505 & 0.0427 & 0.0385 & 0.0373 & 0.0360 \\
\hline $\mathrm{C} 7$ & 0.2381 & 0.1563 & 0.1136 & 0.0806 & 0.0676 & 0.0667 & 0.0515 & 0.0431 & 0.0400 & 0.0373 & 0.0602 & 0.0459 & 0.0391 & 0.0373 & 0.0357 \\
\hline $\mathrm{C} 8$ & 0.5000 & 0.2778 & 0.1786 & 0.1042 & 0.0820 & 0.0538 & 0.0442 & 0.0391 & 0.0376 & 0.0360 & 0.0538 & 0.0442 & 0.0391 & 0.0376 & 0.0360 \\
\hline C9 & 0.0649 & 0.0476 & 0.0397 & 0.0376 & 0.0357 & 0.0420 & 0.0376 & 0.0357 & 0.0357 & 0.0357 & 0.0420 & 0.0376 & 0.0357 & 0.0357 & 0.0357 \\
\hline $\mathrm{C} 10$ & 0.1923 & 0.1250 & 0.0893 & 0.0676 & 0.0581 & 0.0562 & 0.0442 & 0.0385 & 0.0370 & 0.0357 & 0.0725 & 0.0538 & 0.0439 & 0.0403 & 0.0373 \\
\hline $\mathrm{C} 11$ & 1.0000 & 0.4545 & 0.2778 & 0.1351 & 0.1020 & 0.0505 & 0.0427 & 0.0385 & 0.0373 & 0.0360 & 0.0549 & 0.0459 & 0.0403 & 0.0385 & 0.0362 \\
\hline $\mathrm{C} 12$ & 0.3333 & 0.2083 & 0.1389 & 0.0893 & 0.0725 & 0.0538 & 0.0442 & 0.0391 & 0.0376 & 0.0360 & 0.0538 & 0.0442 & 0.0391 & 0.0376 & 0.0360 \\
\hline $\mathrm{C} 13$ & 0.5000 & 0.2632 & 0.1667 & 0.1020 & 0.0820 & 0.0476 & 0.0413 & 0.0379 & 0.0370 & 0.0360 & 0.0495 & 0.0413 & 0.0373 & 0.0365 & 0.0357 \\
\hline C14 & 0.0893 & 0.0667 & 0.0532 & 0.0467 & 0.0417 & 0.0420 & 0.0376 & 0.0357 & 0.0357 & 0.0357 & 0.0667 & 0.0495 & 0.0410 & 0.0385 & 0.0360 \\
\hline $\mathrm{C} 15$ & 0.5000 & 0.2778 & 0.1786 & 0.1042 & 0.0820 & 0.0562 & 0.0442 & 0.0385 & 0.0370 & 0.0357 & 0.0633 & 0.0515 & 0.0439 & 0.0407 & 0.0376 \\
\hline C16 & 1.0000 & 0.4167 & 0.2500 & 0.1250 & 0.0943 & 0.0602 & 0.0459 & 0.0391 & 0.0373 & 0.0357 & 0.0617 & 0.0495 & 0.0424 & 0.0397 & 0.0373 \\
\hline C17 & 0.1389 & 0.0980 & 0.0714 & 0.0562 & 0.0490 & 0.0649 & 0.0495 & 0.0417 & 0.0391 & 0.0370 & 0.0769 & 0.0562 & 0.0455 & 0.0413 & 0.0385 \\
\hline $\mathrm{C} 18$ & 0.3125 & 0.1786 & 0.1250 & 0.0862 & 0.0714 & 0.0602 & 0.0476 & 0.0410 & 0.0388 & 0.0370 & 0.0649 & 0.0495 & 0.0417 & 0.0391 & 0.0370 \\
\hline C19 & 0.2381 & 0.1613 & 0.1190 & 0.0847 & 0.0714 & 0.0617 & 0.0476 & 0.0403 & 0.0382 & 0.0360 & 0.0562 & 0.0459 & 0.0403 & 0.0385 & 0.0370 \\
\hline $\mathrm{C} 20$ & 0.3125 & 0.2000 & 0.1471 & 0.0980 & 0.0806 & 0.0526 & 0.0427 & 0.0379 & 0.0368 & 0.0357 & 0.0562 & 0.0459 & 0.0403 & 0.0385 & 0.0370 \\
\hline $\mathrm{C} 21$ & 1.0000 & 0.3125 & 0.1786 & 0.1042 & 0.0820 & 0.0467 & 0.0400 & 0.0368 & 0.0362 & 0.0357 & 0.0515 & 0.0442 & 0.0397 & 0.0382 & 0.0362 \\
\hline $\mathrm{C} 22$ & 0.0725 & 0.0538 & 0.0439 & 0.0403 & 0.0373 & 0.0704 & 0.0515 & 0.0424 & 0.0394 & 0.0370 & 0.1064 & 0.0820 & 0.0610 & 0.0500 & 0.0427 \\
\hline $\mathrm{C} 23$ & 0.1923 & 0.1316 & 0.0962 & 0.0714 & 0.0610 & 0.0526 & 0.0442 & 0.0397 & 0.0382 & 0.0370 & 0.0649 & 0.0476 & 0.0397 & 0.0376 & 0.0357 \\
\hline $\mathrm{C} 24$ & 0.3125 & 0.1724 & 0.1190 & 0.0820 & 0.0676 & 0.0562 & 0.0442 & 0.0385 & 0.0370 & 0.0357 & 0.1190 & 0.0820 & 0.0610 & 0.0505 & 0.0450 \\
\hline $\mathrm{C} 25$ & 0.2381 & 0.1471 & 0.1042 & 0.0758 & 0.0641 & 0.0649 & 0.0476 & 0.0397 & 0.0376 & 0.0357 & 0.0704 & 0.0538 & 0.0446 & 0.0410 & 0.0385 \\
\hline $\mathrm{C} 26$ & 0.1613 & 0.1111 & 0.0806 & 0.0617 & 0.0532 & 0.0562 & 0.0442 & 0.0385 & 0.0370 & 0.0357 & 0.0943 & 0.0725 & 0.0556 & 0.0472 & 0.0410 \\
\hline $\mathrm{C} 27$ & 0.2500 & 0.1515 & 0.1000 & 0.0704 & 0.0588 & 0.0602 & 0.0476 & 0.0410 & 0.0388 & 0.0370 & 0.0575 & 0.0459 & 0.0397 & 0.0379 & 0.0360 \\
\hline C28 & 0.3333 & 0.2000 & 0.1316 & 0.0847 & 0.0685 & 0.0575 & 0.0476 & 0.0417 & 0.0394 & 0.0373 & 0.0575 & 0.0476 & 0.0417 & 0.0394 & 0.0373 \\
\hline $\mathrm{C} 29$ & 0.2381 & 0.1563 & 0.1136 & 0.0806 & 0.0676 & 0.0538 & 0.0442 & 0.0391 & 0.0376 & 0.0360 & 0.0667 & 0.0515 & 0.0431 & 0.0400 & 0.0373 \\
\hline $\mathrm{C} 30$ & 0.3333 & 0.1923 & 0.1250 & 0.0833 & 0.0685 & 0.0649 & 0.0515 & 0.0439 & 0.0407 & 0.0385 & 0.0617 & 0.0495 & 0.0424 & 0.0397 & 0.0373 \\
\hline
\end{tabular}


Table 6. Cont.

\begin{tabular}{|c|c|c|c|c|c|c|c|c|c|c|}
\hline & \multicolumn{5}{|c|}{ AS4 } & \multicolumn{5}{|c|}{ AS5 } \\
\hline & $x_{i j}$ & $\tilde{\boldsymbol{x}}_{i j}$ & $y_{i j}$ & $\tilde{\boldsymbol{z}}_{i j}$ & $z_{i j}$ & $x_{i j}$ & $\tilde{\boldsymbol{x}}_{i j}$ & $y_{i j}$ & $\tilde{\boldsymbol{z}}_{i j}$ & $z_{i j}$ \\
\hline $\mathrm{C} 1$ & 0.3333 & 0.1852 & 0.1190 & 0.0794 & 0.0649 & 0.2500 & 0.1515 & 0.1000 & 0.0704 & 0.0588 \\
\hline $\mathrm{C} 2$ & 0.2381 & 0.1471 & 0.1042 & 0.0758 & 0.0641 & 0.5000 & 0.2778 & 0.1786 & 0.1042 & 0.0820 \\
\hline $\mathrm{C} 3$ & 0.3333 & 0.1724 & 0.1087 & 0.0746 & 0.0617 & 0.2000 & 0.1282 & 0.0862 & 0.0633 & 0.0538 \\
\hline $\mathrm{C} 4$ & 0.3333 & 0.2083 & 0.1389 & 0.0926 & 0.0769 & 1.0000 & 0.4167 & 0.2500 & 0.1316 & 0.1020 \\
\hline $\mathrm{C} 5$ & 0.2381 & 0.1351 & 0.0926 & 0.0685 & 0.0581 & 0.2381 & 0.1351 & 0.0926 & 0.0685 & 0.0581 \\
\hline C6 & 0.1563 & 0.0980 & 0.0714 & 0.0575 & 0.0505 & 0.3333 & 0.1724 & 0.1087 & 0.0746 & 0.0617 \\
\hline $\mathrm{C} 7$ & 0.1852 & 0.1163 & 0.0833 & 0.0641 & 0.0556 & 0.3333 & 0.1724 & 0.1087 & 0.0746 & 0.0617 \\
\hline $\mathrm{C} 8$ & 0.1042 & 0.0725 & 0.0556 & 0.0476 & 0.0431 & 0.1923 & 0.1220 & 0.0862 & 0.0649 & 0.0556 \\
\hline C9 & 0.0667 & 0.0495 & 0.0410 & 0.0385 & 0.0360 & 0.0649 & 0.0476 & 0.0397 & 0.0376 & 0.0357 \\
\hline $\mathrm{C} 10$ & 0.2381 & 0.1471 & 0.1042 & 0.0758 & 0.0641 & 0.5000 & 0.2500 & 0.1563 & 0.0962 & 0.0769 \\
\hline $\mathrm{C} 11$ & 0.3333 & 0.1724 & 0.1087 & 0.0746 & 0.0617 & 0.1923 & 0.1282 & 0.0926 & 0.0685 & 0.0581 \\
\hline $\mathrm{C} 12$ & 0.2381 & 0.1351 & 0.0926 & 0.0685 & 0.0581 & 0.1613 & 0.1064 & 0.0758 & 0.0588 & 0.0510 \\
\hline $\mathrm{C} 13$ & 0.1923 & 0.1220 & 0.0862 & 0.0649 & 0.0556 & 0.3125 & 0.1724 & 0.1190 & 0.0820 & 0.0676 \\
\hline C14 & 0.0725 & 0.0562 & 0.0455 & 0.0413 & 0.0368 & 0.0769 & 0.0617 & 0.0490 & 0.0435 & 0.0373 \\
\hline $\mathrm{C} 15$ & 0.2381 & 0.1429 & 0.1000 & 0.0725 & 0.0610 & 0.2000 & 0.1220 & 0.0806 & 0.0602 & 0.0515 \\
\hline $\mathrm{C} 16$ & 0.1613 & 0.1020 & 0.0714 & 0.0562 & 0.0490 & 0.3125 & 0.1515 & 0.1000 & 0.0725 & 0.0610 \\
\hline $\mathrm{C} 17$ & 0.2500 & 0.1515 & 0.1000 & 0.0704 & 0.0588 & 0.2500 & 0.1515 & 0.1000 & 0.0704 & 0.0588 \\
\hline C18 & 0.1389 & 0.0943 & 0.0676 & 0.0538 & 0.0472 & 0.2941 & 0.1515 & 0.1042 & 0.0758 & 0.0641 \\
\hline C19 & 0.3125 & 0.1515 & 0.1000 & 0.0725 & 0.0610 & 0.3333 & 0.1724 & 0.1087 & 0.0746 & 0.0617 \\
\hline $\mathrm{C} 20$ & 0.3125 & 0.1515 & 0.1000 & 0.0725 & 0.0610 & 0.2500 & 0.1429 & 0.0926 & 0.0667 & 0.0562 \\
\hline $\mathrm{C} 21$ & 0.3125 & 0.1613 & 0.1087 & 0.0769 & 0.0641 & 0.2381 & 0.1351 & 0.0926 & 0.0685 & 0.0581 \\
\hline $\mathrm{C} 22$ & 1.0000 & 0.2000 & 0.1087 & 0.0746 & 0.0617 & 0.4545 & 0.1515 & 0.0926 & 0.0685 & 0.0581 \\
\hline $\mathrm{C} 23$ & 0.1923 & 0.1220 & 0.0862 & 0.0649 & 0.0556 & 0.3333 & 0.1724 & 0.1087 & 0.0746 & 0.0617 \\
\hline $\mathrm{C} 24$ & 0.1923 & 0.1163 & 0.0806 & 0.0617 & 0.0532 & 0.2381 & 0.1282 & 0.0862 & 0.0649 & 0.0556 \\
\hline $\mathrm{C} 25$ & 0.1923 & 0.1282 & 0.0926 & 0.0685 & 0.0581 & 0.1351 & 0.0943 & 0.0714 & 0.0575 & 0.0505 \\
\hline $\mathrm{C} 26$ & 0.1923 & 0.1282 & 0.0926 & 0.0704 & 0.0610 & 0.3333 & 0.1724 & 0.1087 & 0.0746 & 0.0617 \\
\hline $\mathrm{C} 27$ & 0.5000 & 0.1852 & 0.1087 & 0.0746 & 0.0617 & 0.3125 & 0.1515 & 0.1000 & 0.0725 & 0.0610 \\
\hline $\mathrm{C} 28$ & 0.1923 & 0.1220 & 0.0862 & 0.0649 & 0.0556 & 0.1852 & 0.1111 & 0.0806 & 0.0633 & 0.0549 \\
\hline C29 & 0.3333 & 0.1852 & 0.1190 & 0.0794 & 0.0649 & 0.3333 & 0.1613 & 0.1000 & 0.0704 & 0.0588 \\
\hline $\mathrm{C} 30$ & 0.2000 & 0.1351 & 0.0926 & 0.0667 & 0.0562 & 0.2381 & 0.1351 & 0.0926 & 0.0685 & 0.0581 \\
\hline
\end{tabular}


(2) Table 7 presents the interval value by adopting Equation (3). However, it generated the information lost during the computation; thus, the interval value should be addressed as a crisp value using Equation (4). Therefore, Table 8 expresses the grey relational coefficient generated from Equation (5).

Table 7. Interval value $\vartheta_{i j}^{*}$.

\begin{tabular}{|c|c|c|c|c|c|c|c|c|c|c|}
\hline & \multicolumn{2}{|c|}{$\left[\widehat{\vartheta}_{1 j}, \breve{\vartheta}_{1 j}\right]$} & \multicolumn{2}{|c|}{$\left[\widehat{\boldsymbol{\vartheta}}_{2 j}, \breve{\boldsymbol{\vartheta}}_{2 j}\right]$} & \multicolumn{2}{|c|}{$\left[\widehat{\boldsymbol{\vartheta}}_{3 j}, \breve{\boldsymbol{\vartheta}}_{3 j}\right]$} & \multicolumn{2}{|c|}{$\left[\widehat{\boldsymbol{\vartheta}}_{4 j}, \overline{\boldsymbol{\vartheta}}_{4 j}\right]$} & \multicolumn{2}{|c|}{$\left[\widehat{\boldsymbol{\vartheta}}_{5 j}, \breve{\boldsymbol{\vartheta}}_{5 j}\right]$} \\
\hline $\mathrm{C} 1$ & 0.0629 & 0.0545 & 0.0562 & 0.0036 & 0.0380 & 0.0069 & 0.0688 & 0.0595 & 0.0697 & 0.0419 \\
\hline $\mathrm{C} 2$ & 0.0659 & 0.0663 & 0.0476 & 0.0048 & 0.0375 & 0.0068 & 0.0671 & 0.0394 & 0.0564 & 0.0810 \\
\hline $\mathrm{C} 3$ & 0.0614 & 0.1786 & 0.0199 & 0.0036 & 0.0182 & 0.0037 & 0.0744 & 0.0636 & 0.0698 & 0.0316 \\
\hline $\mathrm{C} 4$ & 0.0667 & 0.0429 & 0.1012 & 0.0088 & 0.0625 & 0.0138 & 0.0544 & 0.0516 & 0.0497 & 0.1131 \\
\hline $\mathrm{C} 5$ & 0.0614 & 0.1764 & 0.0085 & 0.0045 & 0.0136 & 0.0030 & 0.0768 & 0.0434 & 0.0768 & 0.0434 \\
\hline C6 & 0.0550 & 0.2593 & 0.0216 & 0.0029 & 0.0182 & 0.0037 & 0.0774 & 0.0257 & 0.0744 & 0.0636 \\
\hline C7 & 0.0616 & 0.0809 & 0.0258 & 0.0072 & 0.0352 & 0.0065 & 0.0727 & 0.0303 & 0.0744 & 0.0636 \\
\hline $\mathrm{C} 8$ & 0.0564 & 0.1783 & 0.0236 & 0.0045 & 0.0236 & 0.0045 & 0.0670 & 0.0145 & 0.0718 & 0.0311 \\
\hline C9 & 0.0424 & 0.0100 & 0.0057 & 0.0018 & 0.0057 & 0.0018 & 0.0433 & 0.0080 & 0.0424 & 0.0078 \\
\hline $\mathrm{C} 10$ & 0.0678 & 0.0647 & 0.0637 & 0.0054 & 0.0487 & 0.0088 & 0.0671 & 0.0394 & 0.0624 & 0.0894 \\
\hline $\mathrm{C} 11$ & 0.0489 & 0.2565 & 0.0290 & 0.0037 & 0.0242 & 0.0046 & 0.0744 & 0.0636 & 0.0669 & 0.0291 \\
\hline $\mathrm{C} 12$ & 0.0584 & 0.1230 & 0.0236 & 0.0045 & 0.0236 & 0.0045 & 0.0768 & 0.0434 & 0.0712 & 0.0247 \\
\hline $\mathrm{C} 13$ & 0.0574 & 0.1811 & 0.0180 & 0.0030 & 0.0175 & 0.0036 & 0.0718 & 0.0311 & 0.0706 & 0.0573 \\
\hline C14 & 0.0589 & 0.0233 & 0.0585 & 0.0018 & 0.0433 & 0.0080 & 0.0453 & 0.0084 & 0.0456 & 0.0087 \\
\hline $\mathrm{C} 15$ & 0.0564 & 0.1776 & 0.0450 & 0.0054 & 0.0345 & 0.0060 & 0.0716 & 0.0409 & 0.0745 & 0.0336 \\
\hline C16 & 0.0542 & 0.2517 & 0.0386 & 0.0065 & 0.0340 & 0.0059 & 0.0754 & 0.0261 & 0.0817 & 0.0639 \\
\hline $\mathrm{C} 17$ & 0.0656 & 0.0357 & 0.0649 & 0.0071 & 0.0529 & 0.0095 & 0.0697 & 0.0419 & 0.0697 & 0.0419 \\
\hline $\mathrm{C} 18$ & 0.0658 & 0.1123 & 0.0442 & 0.0058 & 0.0404 & 0.0071 & 0.0695 & 0.0203 & 0.0771 & 0.0577 \\
\hline C19 & 0.0568 & 0.0851 & 0.0228 & 0.0066 & 0.0270 & 0.0048 & 0.0817 & 0.0639 & 0.0744 & 0.0636 \\
\hline $\mathrm{C} 20$ & 0.0549 & 0.1201 & 0.0312 & 0.0044 & 0.0270 & 0.0048 & 0.0817 & 0.0639 & 0.0749 & 0.0447 \\
\hline $\mathrm{C} 21$ & 0.0663 & 0.2555 & 0.0240 & 0.0029 & 0.0188 & 0.0038 & 0.0762 & 0.0608 & 0.0768 & 0.0434 \\
\hline $\mathrm{C} 22$ & 0.0487 & 0.0012 & 0.1036 & 0.0086 & 0.0582 & 0.0127 & 0.0950 & 0.1767 & 0.0974 & 0.1083 \\
\hline $\mathrm{C} 23$ & 0.0626 & 0.0671 & 0.0503 & 0.0039 & 0.0424 & 0.0078 & 0.0718 & 0.0311 & 0.0744 & 0.0636 \\
\hline $\mathrm{C} 24$ & 0.0706 & 0.1131 & 0.1241 & 0.0054 & 0.0689 & 0.0170 & 0.0766 & 0.0329 & 0.0819 & 0.0456 \\
\hline $\mathrm{C} 25$ & 0.0671 & 0.0809 & 0.0538 & 0.0078 & 0.0450 & 0.0078 & 0.0669 & 0.0291 & 0.0676 & 0.0190 \\
\hline $\mathrm{C} 26$ & 0.0667 & 0.0507 & 0.0979 & 0.0054 & 0.0564 & 0.0113 & 0.0635 & 0.0287 & 0.0744 & 0.0636 \\
\hline $\mathrm{C} 27$ & 0.0697 & 0.0845 & 0.0266 & 0.0058 & 0.0296 & 0.0055 & 0.0846 & 0.1097 & 0.0817 & 0.0639 \\
\hline $\mathrm{C} 28$ & 0.0631 & 0.1192 & 0.0276 & 0.0048 & 0.0276 & 0.0048 & 0.0718 & 0.0311 & 0.0785 & 0.0322 \\
\hline $\mathrm{C} 29$ & 0.0616 & 0.0871 & 0.0532 & 0.0045 & 0.0411 & 0.0072 & 0.0688 & 0.0595 & 0.0798 & 0.0672 \\
\hline $\mathrm{C} 30$ & 0.0642 & 0.1155 & 0.0298 & 0.0063 & 0.0340 & 0.0059 & 0.0648 & 0.0293 & 0.0768 & 0.0434 \\
\hline & \multicolumn{2}{|c|}{$\left[\bar{\vartheta}_{\text {max }}, \tilde{\vartheta}_{\text {max }}\right]$} & 0.1651 & 0.4306 & & & & & & \\
\hline & \multicolumn{2}{|c|}{$\left[\bar{\vartheta}_{\min }, \tilde{\vartheta}_{\min }\right]$} & 0.0160 & 0.0094 & & & & & & \\
\hline
\end{tabular}


Table 8. Grey relational coefficient.

\begin{tabular}{|c|c|c|c|c|c|c|c|c|c|c|}
\hline & \multicolumn{2}{|c|}{$\left[\varepsilon_{1 j}^{\prime}, \varepsilon_{1 j}^{*}\right]$} & \multicolumn{2}{|c|}{$\left[\varepsilon_{2 j}^{\prime}, \varepsilon_{2 j}^{*}\right]$} & \multicolumn{2}{|c|}{$\left[\varepsilon_{3 \mathbf{j}}^{\prime}, \varepsilon_{3 \mathrm{j}}^{*}\right]$} & \multicolumn{2}{|c|}{$\left[\varepsilon_{4 \mathbf{j}}^{\prime}, \varepsilon_{4 \mathrm{j}}^{*}\right]$} & \multicolumn{2}{|c|}{$\left[\varepsilon_{5 j}^{\prime}, \varepsilon_{5 j}^{*}\right]$} \\
\hline $\mathrm{C} 1$ & 0.5425 & 0.7103 & 0.5734 & 0.9816 & 0.6777 & 0.9578 & 0.5182 & 0.6916 & 0.5147 & 0.7628 \\
\hline $\mathrm{C} 2$ & 0.5299 & 0.6678 & 0.6186 & 0.9734 & 0.6807 & 0.9585 & 0.5249 & 0.7740 & 0.5722 & 0.6211 \\
\hline $\mathrm{C} 3$ & 0.5491 & 0.4245 & 0.8273 & 0.9816 & 0.8444 & 0.9811 & 0.4970 & 0.6771 & 0.5143 & 0.8116 \\
\hline $\mathrm{C} 4$ & 0.5266 & 0.7582 & 0.4154 & 0.9452 & 0.5445 & 0.9119 & 0.5822 & 0.7217 & 0.6065 & 0.5389 \\
\hline C5 & 0.5491 & 0.4275 & 0.9608 & 0.9751 & 0.8965 & 0.9864 & 0.4882 & 0.7563 & 0.4882 & 0.7563 \\
\hline C6 & 0.5791 & 0.3364 & 0.8105 & 0.9867 & 0.8444 & 0.9811 & 0.4863 & 0.8422 & 0.4970 & 0.6771 \\
\hline C7 & 0.5484 & 0.6213 & 0.7717 & 0.9560 & 0.6968 & 0.9612 & 0.5030 & 0.8182 & 0.4970 & 0.6771 \\
\hline C8 & 0.5722 & 0.4248 & 0.7918 & 0.9751 & 0.7918 & 0.9751 & 0.5252 & 0.9075 & 0.5064 & 0.8140 \\
\hline $\mathrm{C} 9$ & 0.6492 & 0.9370 & 1.0000 & 0.9953 & 1.0000 & 0.9953 & 0.6433 & 0.9508 & 0.6492 & 0.9521 \\
\hline $\mathrm{C} 10$ & 0.5221 & 0.6732 & 0.5393 & 0.9690 & 0.6124 & 0.9452 & 0.5249 & 0.7740 & 0.5448 & 0.5972 \\
\hline C11 & 0.6108 & 0.3388 & 0.7445 & 0.9811 & 0.7858 & 0.9746 & 0.4970 & 0.6771 & 0.5260 & 0.8243 \\
\hline C12 & 0.5629 & 0.5178 & 0.7918 & 0.9751 & 0.7918 & 0.9751 & 0.4882 & 0.7563 & 0.5089 & 0.8477 \\
\hline $\mathrm{C} 13$ & 0.5678 & 0.4210 & 0.8467 & 0.9864 & 0.8518 & 0.9816 & 0.5064 & 0.8140 & 0.5112 & 0.6997 \\
\hline $\mathrm{C} 14$ & 0.5604 & 0.8557 & 0.5625 & 0.9953 & 0.6433 & 0.9508 & 0.6316 & 0.9475 & 0.6296 & 0.9460 \\
\hline $\mathrm{C} 15$ & 0.5722 & 0.4258 & 0.6333 & 0.9690 & 0.7022 & 0.9645 & 0.5073 & 0.7672 & 0.4965 & 0.8014 \\
\hline $\mathrm{C} 16$ & 0.5834 & 0.3431 & 0.6733 & 0.9612 & 0.7058 & 0.9651 & 0.4932 & 0.8399 & 0.4717 & 0.6760 \\
\hline C17 & 0.5312 & 0.7913 & 0.5340 & 0.9570 & 0.5898 & 0.9401 & 0.5147 & 0.7628 & 0.5147 & 0.7628 \\
\hline $\mathrm{C} 18$ & 0.5304 & 0.5407 & 0.6379 & 0.9658 & 0.6620 & 0.9570 & 0.5154 & 0.8727 & 0.4871 & 0.6984 \\
\hline C19 & 0.5704 & 0.6091 & 0.7989 & 0.9602 & 0.7617 & 0.9734 & 0.4717 & 0.6760 & 0.4970 & 0.6771 \\
\hline C20 & 0.5796 & 0.5239 & 0.7266 & 0.9757 & 0.7617 & 0.9734 & 0.4717 & 0.6760 & 0.4952 & 0.7505 \\
\hline $\mathrm{C} 21$ & 0.5282 & 0.3396 & 0.7876 & 0.9867 & 0.8383 & 0.9807 & 0.4905 & 0.6869 & 0.4882 & 0.7563 \\
\hline $\mathrm{C} 22$ & 0.6124 & 1.0000 & 0.4094 & 0.9466 & 0.5637 & 0.9189 & 0.4318 & 0.4270 & 0.4253 & 0.5497 \\
\hline $\mathrm{C} 23$ & 0.5438 & 0.6650 & 0.6033 & 0.9799 & 0.6492 & 0.9521 & 0.5064 & 0.8140 & 0.4970 & 0.6771 \\
\hline $\mathrm{C} 24$ & 0.5112 & 0.5390 & 0.3641 & 0.9690 & 0.5179 & 0.8923 & 0.4889 & 0.8048 & 0.4710 & 0.7466 \\
\hline $\mathrm{C} 25$ & 0.5249 & 0.6214 & 0.5850 & 0.9521 & 0.6336 & 0.9522 & 0.5260 & 0.8243 & 0.5230 & 0.8802 \\
\hline $\mathrm{C} 26$ & 0.5266 & 0.7254 & 0.4240 & 0.9690 & 0.5724 & 0.9282 & 0.5399 & 0.8262 & 0.4970 & 0.6771 \\
\hline $\mathrm{C} 27$ & 0.5147 & 0.6110 & 0.7648 & 0.9658 & 0.7401 & 0.9682 & 0.4624 & 0.5466 & 0.4717 & 0.6760 \\
\hline $\mathrm{C} 28$ & 0.5416 & 0.5257 & 0.7566 & 0.9729 & 0.7566 & 0.9729 & 0.5064 & 0.8140 & 0.4825 & 0.8083 \\
\hline C29 & 0.5484 & 0.6037 & 0.5881 & 0.9751 & 0.6576 & 0.9560 & 0.5182 & 0.6916 & 0.4780 & 0.6648 \\
\hline C 30 & 0.5372 & 0.5337 & 0.7378 & 0.9623 & 0.7058 & 0.9651 & 0.5343 & 0.8231 & 0.4882 & 0.7563 \\
\hline
\end{tabular}

(3) Before converting the grey relational coefficient into the grey relational grade, the weightage vector must be obtained first. Hence, according to Equation (6), the weights are:

$$
\begin{array}{llllll}
v_{1}=0.0331 & v_{6}=0.0336 & v_{11}=0.0332 & v_{16}=0.0320 & v_{21}=0.0328 & v_{26}=0.0319 \\
v_{2}=0.0330 & v_{7}=0.0336 & v_{12}=0.0344 & v_{17}=0.0329 & v_{22}=0.0300 & v_{27}=0.0321 \\
v_{3}=0.0339 & v_{8}=0.0347 & v_{13}=0.0343 & v_{18}=0.0328 & v_{23}=0.0329 & v_{28}=0.0340 \\
v_{4}=0.0313 & v_{9}=0.0418 & v_{14}=0.0368 & v_{19}=0.0334 & v_{24}=0.0301 & v_{29}=0.0319 \\
v_{5}=0.0347 & v_{10}=0.0320 & v_{15}=0.0326 & v_{20}=0.0331 & v_{25}=0.0335 & v_{30}=0.0336
\end{array}
$$

Next, insert the collected weights into Equation (7), for which the grey relational grades are formulated in Table 9.

(4) Applying Equations (8) and (9), the likelihood relationship of $A S 1 \geqslant A S 2$ can be calculated as follows: $\left(D_{A S 1} \geqslant D_{A S 2}\right)=\max \left\{1-\max \left\{\left(\tilde{d}_{A S 2}-\bar{d}_{A S 1}\right) /\left(\varphi\left(D_{A S 1}\right)+\varphi\left(D_{A S 2}\right)\right), 0\right\}, 0\right\}=$ $\max \{1-\max \{(0.972-0.555) /((0.555-0.581)+(0.972-0.683)), 0\}, 0\}=0$. 
Table 9. Grey relational grade.

\begin{tabular}{|c|c|c|c|c|c|c|c|c|c|c|}
\hline & \multicolumn{2}{|c|}{$\left[\bar{d}_{1}, \widetilde{d}_{1}\right]$} & \multicolumn{2}{|c|}{$\left[\overline{\boldsymbol{d}}_{2}, \widetilde{\boldsymbol{d}}_{2}\right]$} & \multicolumn{2}{|c|}{$\left[\bar{d}_{3}, d_{3}\right]$} & \multicolumn{2}{|c|}{$\left[\bar{d}_{4}, \widetilde{d}_{4}\right]$} & \multicolumn{2}{|c|}{$\left[\bar{d}_{5}, \widetilde{d}_{5}\right]$} \\
\hline $\mathrm{C} 1$ & 0.0179 & 0.0235 & 0.0190 & 0.0325 & 0.0224 & 0.0317 & 0.0171 & 0.0229 & 0.0170 & 0.0252 \\
\hline $\mathrm{C} 2$ & 0.0175 & 0.0220 & 0.0204 & 0.0321 & 0.0225 & 0.0316 & 0.0173 & 0.0256 & 0.0189 & 0.0205 \\
\hline $\mathrm{C} 3$ & 0.0186 & 0.0144 & 0.0281 & 0.0333 & 0.0286 & 0.0333 & 0.0169 & 0.0230 & 0.0174 & 0.0275 \\
\hline $\mathrm{C} 4$ & 0.0165 & 0.0237 & 0.0130 & 0.0295 & 0.0170 & 0.0285 & 0.0182 & 0.0226 & 0.0190 & 0.0168 \\
\hline $\mathrm{C} 5$ & 0.0191 & 0.0149 & 0.0334 & 0.0339 & 0.0312 & 0.0343 & 0.0170 & 0.0263 & 0.0170 & 0.0263 \\
\hline C6 & 0.0194 & 0.0113 & 0.0272 & 0.0331 & 0.0284 & 0.0330 & 0.0163 & 0.0283 & 0.0167 & 0.0227 \\
\hline C7 & 0.0184 & 0.0209 & 0.0260 & 0.0322 & 0.0234 & 0.0323 & 0.0169 & 0.0275 & 0.0167 & 0.0228 \\
\hline $\mathrm{C} 8$ & 0.0199 & 0.0148 & 0.0275 & 0.0339 & 0.0275 & 0.0339 & 0.0182 & 0.0315 & 0.0176 & 0.0283 \\
\hline C9 & 0.0272 & 0.0392 & 0.0418 & 0.0417 & 0.0418 & 0.0417 & 0.0269 & 0.0398 & 0.0272 & 0.0398 \\
\hline $\mathrm{C} 10$ & 0.0167 & 0.0215 & 0.0172 & 0.0310 & 0.0196 & 0.0302 & 0.0168 & 0.0247 & 0.0174 & 0.0191 \\
\hline $\mathrm{C} 11$ & 0.0203 & 0.0112 & 0.0247 & 0.0326 & 0.0261 & 0.0324 & 0.0165 & 0.0225 & 0.0175 & 0.0274 \\
\hline $\mathrm{C} 12$ & 0.0194 & 0.0178 & 0.0273 & 0.0336 & 0.0273 & 0.0336 & 0.0168 & 0.0260 & 0.0175 & 0.0292 \\
\hline $\mathrm{C} 13$ & 0.0195 & 0.0144 & 0.0290 & 0.0338 & 0.0292 & 0.0337 & 0.0174 & 0.0279 & 0.0175 & 0.0240 \\
\hline C14 & 0.0206 & 0.0315 & 0.0207 & 0.0367 & 0.0237 & 0.0350 & 0.0233 & 0.0349 & 0.0232 & 0.0348 \\
\hline $\mathrm{C} 15$ & 0.0187 & 0.0139 & 0.0207 & 0.0316 & 0.0229 & 0.0315 & 0.0166 & 0.0250 & 0.0162 & 0.0261 \\
\hline $\mathrm{C} 16$ & 0.0187 & 0.0110 & 0.0216 & 0.0308 & 0.0226 & 0.0309 & 0.0158 & 0.0269 & 0.0151 & 0.0216 \\
\hline $\mathrm{C} 17$ & 0.0175 & 0.0260 & 0.0176 & 0.0315 & 0.0194 & 0.0309 & 0.0169 & 0.0251 & 0.0169 & 0.0251 \\
\hline $\mathrm{C} 18$ & 0.0174 & 0.0177 & 0.0209 & 0.0316 & 0.0217 & 0.0314 & 0.0169 & 0.0286 & 0.0160 & 0.0229 \\
\hline C19 & 0.0190 & 0.0203 & 0.0267 & 0.0320 & 0.0254 & 0.0325 & 0.0157 & 0.0226 & 0.0166 & 0.0226 \\
\hline $\mathrm{C} 20$ & 0.0192 & 0.0173 & 0.0240 & 0.0323 & 0.0252 & 0.0322 & 0.0156 & 0.0224 & 0.0164 & 0.0248 \\
\hline $\mathrm{C} 21$ & 0.0173 & 0.0112 & 0.0259 & 0.0324 & 0.0275 & 0.0322 & 0.0161 & 0.0226 & 0.0160 & 0.0248 \\
\hline $\mathrm{C} 22$ & 0.0184 & 0.0300 & 0.0123 & 0.0284 & 0.0169 & 0.0275 & 0.0129 & 0.0128 & 0.0128 & 0.0165 \\
\hline $\mathrm{C} 23$ & 0.0179 & 0.0219 & 0.0198 & 0.0322 & 0.0213 & 0.0313 & 0.0166 & 0.0267 & 0.0163 & 0.0222 \\
\hline $\mathrm{C} 24$ & 0.0154 & 0.0162 & 0.0110 & 0.0291 & 0.0156 & 0.0268 & 0.0147 & 0.0242 & 0.0142 & 0.0225 \\
\hline $\mathrm{C} 25$ & 0.0176 & 0.0208 & 0.0196 & 0.0319 & 0.0212 & 0.0319 & 0.0176 & 0.0276 & 0.0175 & 0.0295 \\
\hline $\mathrm{C} 26$ & 0.0168 & 0.0231 & 0.0135 & 0.0309 & 0.0183 & 0.0296 & 0.0172 & 0.0263 & 0.0159 & 0.0216 \\
\hline $\mathrm{C} 27$ & 0.0165 & 0.0196 & 0.0245 & 0.0310 & 0.0237 & 0.0310 & 0.0148 & 0.0175 & 0.0151 & 0.0217 \\
\hline $\mathrm{C} 28$ & 0.0184 & 0.0179 & 0.0258 & 0.0331 & 0.0258 & 0.0331 & 0.0172 & 0.0277 & 0.0164 & 0.0275 \\
\hline $\mathrm{C} 29$ & 0.0175 & 0.0192 & 0.0187 & 0.0311 & 0.0210 & 0.0305 & 0.0165 & 0.0220 & 0.0152 & 0.0212 \\
\hline C30 & 0.0181 & 0.0179 & 0.0248 & 0.0323 & 0.0237 & 0.0324 & 0.0180 & 0.0277 & 0.0164 & 0.0254 \\
\hline
\end{tabular}

Afterward, the likelihood relationship can be arranged into a likelihood matrix using Equation (10), and the matrix is then decomposed using MATLAB 10 to obtain the eigenvectors. The higher eigenvector has greater importance in the SBMCC.

Table 10 expresses the likelihood matrix of the aspects, which includes five eigenvectors 0.0983 , 0.6393, 0.6758, 0.2725, and 0.2252. Thus, the likelihood relation for the SBMCC aspects can be represented as $A S_{3} \geqslant A S_{2} \geqslant A S_{4} \geqslant A S_{5} \geqslant A S_{1}$. This reveals that technology capabilities (AS3) are given the highest priority, followed by networking and social capabilities (AS2), and capabilities for learning and developing (AS4).

Table 11 indicates that the top-five influential criteria are developing social relations with unfamiliar actors inside or outside the organization for information gathering, experimentation and negotiation (C9), upgrading and integrating technology capabilities, new product development and marketing (C14), the competency to think inventively (C5), building trust, a shared vision and agreement on basic values 
(C8), and new approaches in the knowledge for performing management functions and new processes that produce changes in the organization's strategy, structure, administrative procedures, and systems (C13). The ranking of criteria can be stated as: $C_{9} \geqslant C_{14} \geqslant C_{5} \geqslant C_{8} \geqslant C_{13} \geqslant C_{12} \geqslant C_{28} \geqslant C_{3} \geqslant C_{7} \geqslant$ $\mathrm{C}_{6} \geqslant \mathrm{C}_{30} \geqslant \mathrm{C}_{25} \geqslant \mathrm{C}_{19} \geqslant \mathrm{C}_{11} \geqslant \mathrm{C}_{1} \geqslant \mathrm{C}_{20} \geqslant \mathrm{C}_{2} \geqslant \mathrm{C}_{17} \geqslant \mathrm{C}_{23} \geqslant \mathrm{C}_{18} \geqslant \mathrm{C}_{21} \geqslant \mathrm{C}_{15} \geqslant \mathrm{C}_{26} \geqslant \mathrm{C}_{10} \geqslant$ $\mathrm{C}_{29} \geqslant \mathrm{C}_{27} \geqslant \mathrm{C}_{16} \geqslant \mathrm{C}_{4} \geqslant \mathrm{C}_{24} \geqslant \mathrm{C}_{22}$. These results provide significant evidence and a quantitative basis for the case firm to understand innovation in current SBMCC practice.

Table 10. Likelihood matrix for aspects.

\begin{tabular}{cccccccc}
\hline & AS1 & AS2 & AS3 & AS4 & AS5 & Eigenvectors & Ranking \\
\hline AS1 & 0.500 & 0.000 & 0.000 & 0.248 & 0.279 & 0.0983 & 5 \\
AS2 & 1.000 & 0.500 & 0.474 & 0.841 & 0.888 & 0.6393 & 2 \\
AS3 & 1.000 & 0.526 & 0.500 & 0.902 & 0.958 & 0.6758 & 1 \\
AS4 & 0.752 & 0.159 & 0.098 & 0.500 & 0.531 & 0.2725 & 3 \\
AS5 & 0.721 & 0.112 & 0.042 & 0.469 & 0.500 & 0.2252 & 4 \\
\hline
\end{tabular}

Table 11. Likelihood matrix for criteria.

\begin{tabular}{|c|c|c|c|c|c|c|c|c|c|c|c|c|c|c|c|}
\hline C1 & $\mathrm{C} 2$ & C3 & C4 & C5 & C6 & C7 & C8 & C9 & $\mathrm{C10}$ & C11 & $\mathrm{C} 12$ & $\mathrm{C13}$ & $\mathrm{C} 14$ & C15 & $\mathrm{C} 16$ \\
\hline
\end{tabular}

$\begin{array}{llllllllllllllllll}\mathrm{C} 1 & 0.5000 & 0.50413 & 0.4073 & 0.6530 & 0.3011 & 0.4414 & 0.4477 & 0.3378 & 0.0000 & 0.5917 & 0.4846 & 0.3704 & 0.3642 & 0.2330 & 0.5398 & 0.6017\end{array}$

$\begin{array}{llllllllllllllllll}\mathrm{C} 2 & 0.4959 & 0.5000 & 0.3904 & 0.6633 & 0.2691 & 0.4283 & 0.4378 & 0.3161 & 0.0000 & 0.5960 & 0.4770 & 0.3523 & 0.3418 & 0.2105 & 0.5392 & 0.6079\end{array}$

$\begin{array}{lllllllllllllllllll}\mathrm{C} 3 & 0.5927 & 0.6096 & 0.5000 & 0.8058 & 0.3482 & 0.5538 & 0.5346 & 0.3869 & 0.0000 & 0.7204 & 0.6160 & 0.4317 & 0.4377 & 0.2388 & 0.6626 & 0.7643\end{array}$

$\begin{array}{llllllllllllllllll}\text { C4 } & 0.3470 & 0.3367 & 0.1942 & 0.5000 & 0.0644 & 0.2257 & 0.2740 & 0.1501 & 0.0000 & 0.4375 & 0.2747 & 0.1859 & 0.1454 & 0.0969 & 0.3698 & 0.4213\end{array}$

$\begin{array}{lllllllllllllllll}\text { C5 } & 0.6989 & 0.7309 & 0.6518 & 0.9356 & 0.5000 & 0.7167 & 0.6530 & 0.5002 & 0.0000 & 0.8415 & 0.7825 & 0.5475 & 0.5859 & 0.3025 & 0.7928 & 0.9194\end{array}$

$\begin{array}{llllllllllllllllllll}\text { C6 } & 0.5586 & 0.5717 & 0.4462 & 0.7743 & 0.2833 & 0.5000 & 0.4939 & 0.3401 & 0.0000 & 0.6875 & 0.5652 & 0.3864 & 0.3810 & 0.2064 & 0.6246 & 0.7250\end{array}$

$\begin{array}{llllllllllllllllllll}\text { C7 } & 0.5523 & 0.5622 & 0.4654 & 0.7260 & 0.3470 & 0.5061 & 0.5000 & 0.3787 & 0.0000 & 0.6565 & 0.5549 & 0.4151 & 0.4166 & 0.2524 & 0.6039 & 0.6799\end{array}$

$\begin{array}{lllllllllllllllll}\mathrm{C} 8 & 0.6622 & 0.6839 & 0.6131 & 0.8499 & 0.4998 & 0.6599 & 0.6213 & 0.5000 & 0.0000 & 0.7756 & 0.7093 & 0.5372 & 0.5636 & 0.3312 & 0.7313 & 0.8229\end{array}$

$\begin{array}{llllllllllllllllllll}\text { C9 } & 1.0000 & 1.0000 & 1.0000 & 1.0000 & 1.0000 & 1.0000 & 1.0000 & 1.0000 & 0.5000 & 1.0000 & 1.0000 & 1.0000 & 1.0000 & 0.9186 & 1.0000 & 1.0000\end{array}$

$\begin{array}{llllllllllllllllllll}\mathrm{C} 10 & 0.4083 & 0.4040 & 0.2796 & 0.5625 & 0.1585 & 0.3125 & 0.3435 & 0.2244 & 0.0000 & 0.5000 & 0.3595 & 0.2592 & 0.2327 & 0.1498 & 0.4384 & 0.4949\end{array}$

$\begin{array}{lllllllllllllllllll}\mathrm{C} 11 & 0.5154 & 0.5230 & 0.3840 & 0.7253 & 0.2175 & 0.4348 & 0.4451 & 0.2907 & 0.0000 & 0.6405 & 0.5000 & 0.3368 & 0.3188 & 0.1759 & 0.5734 & 0.6665\end{array}$

$\begin{array}{lllllllllllllllllll}\mathrm{C} 12 & 0.6296 & 0.6477 & 0.5683 & 0.8141 & 0.4525 & 0.6136 & 0.5849 & 0.4628 & 0.0000 & 0.7408 & 0.6632 & 0.5000 & 0.5187 & 0.3065 & 0.6937 & 0.7811\end{array}$

$\begin{array}{llllllllllllllllll}\mathrm{C} 13 & 0.6358 & 0.6582 & 0.5623 & 0.8546 & 0.4141 & 0.6190 & 0.5834 & 0.4364 & 0.0000 & 0.7673 & 0.6812 & 0.4813 & 0.5000 & 0.2693 & 0.7137 & 0.8227\end{array}$

$\begin{array}{lllllllllllllllllll}\mathrm{C} 14 & 0.7670 & 0.7895 & 0.7612 & 0.9031 & 0.6975 & 0.7936 & 0.7476 & 0.6688 & 0.0814 & 0.8502 & 0.8241 & 0.6935 & 0.7307 & 0.5000 & 0.8244 & 0.8911\end{array}$

$\begin{array}{llllllllllllllllllll}\mathrm{C} 15 & 0.4602 & 0.4608 & 0.3374 & 0.6302 & 0.2072 & 0.3754 & 0.3961 & 0.2687 & 0.0000 & 0.5616 & 0.4266 & 0.3063 & 0.2863 & 0.1756 & 0.5000 & 0.5675\end{array}$

$\begin{array}{lllllllllllllllllllll}\mathrm{C} 16 & 0.3983 & 0.3921 & 0.2357 & 0.5787 & 0.0806 & 0.2750 & 0.3201 & 0.1771 & 0.0000 & 0.5051 & 0.3335 & 0.2189 & 0.1773 & 0.1089 & 0.4325 & 0.5000\end{array}$

$\begin{array}{lllllllllllllllllllll}\mathrm{C} 17 & 0.4885 & 0.4913 & 0.4029 & 0.6268 & 0.3090 & 0.4330 & 0.4401 & 0.3408 & 0.0000 & 0.5714 & 0.4714 & 0.3702 & 0.3646 & 0.2428 & 0.5232 & 0.5775\end{array}$

$\begin{array}{llllllllllllllllllll}\mathrm{C} 18 & 0.4745 & 0.4764 & 0.3689 & 0.6317 & 0.2548 & 0.4037 & 0.4173 & 0.3018 & 0.0000 & 0.5684 & 0.4494 & 0.3360 & 0.3233 & 0.2047 & 0.5127 & 0.5749\end{array}$

$\begin{array}{lllllllllllllllll}\mathrm{C} 19 & 0.5313 & 0.5400 & 0.4218 & 0.7241 & 0.2795 & 0.4677 & 0.4696 & 0.3311 & 0.0000 & 0.6466 & 0.5249 & 0.3725 & 0.3647 & 0.2099 & 0.5863 & 0.6711\end{array}$

$\begin{array}{llllllllllllllllll}\mathrm{C} 20 & 0.5017 & 0.5069 & 0.3846 & 0.6864 & 0.2455 & 0.4275 & 0.4382 & 0.3030 & 0.0000 & 0.6120 & 0.4828 & 0.3432 & 0.3294 & 0.1939 & 0.5505 & 0.6286\end{array}$

$\begin{array}{lllllllllllllllllll}\mathrm{C} 21 & 0.4749 & 0.4775 & 0.3217 & 0.6841 & 0.1460 & 0.3711 & 0.3978 & 0.2388 & 0.0000 & 0.5992 & 0.4384 & 0.2859 & 0.2545 & 0.1420 & 0.5268 & 0.6157\end{array}$

$\begin{array}{llllllllllllllllllll}\mathrm{C} 22 & 0.2582 & 0.2406 & 0.0878 & 0.3975 & 0.0000 & 0.1145 & 0.1802 & 0.0604 & 0.0000 & 0.3401 & 0.1611 & 0.0946 & 0.0414 & 0.0354 & 0.2690 & 0.3090\end{array}$

$\begin{array}{lllllllllllllllll}\mathrm{C} 23 & 0.4834 & 0.4860 & 0.3855 & 0.6352 & 0.2781 & 0.4190 & 0.4294 & 0.3189 & 0.0000 & 0.5742 & 0.4624 & 0.3517 & 0.3422 & 0.2197 & 0.5211 & 0.5814\end{array}$

$\begin{array}{lllllllllllllllllll}\mathrm{C} 24 & 0.2813 & 0.2669 & 0.1325 & 0.4119 & 0.0199 & 0.1578 & 0.2113 & 0.1018 & 0.0000 & 0.3583 & 0.1999 & 0.1331 & 0.0906 & 0.0669 & 0.2938 & 0.3324\end{array}$

$\begin{array}{llllllllllllllllllll}\mathrm{C} 25 & 0.5338 & 0.5405 & 0.4588 & 0.6781 & 0.3651 & 0.4911 & 0.4885 & 0.3881 & 0.0000 & 0.6206 & 0.5301 & 0.4182 & 0.4198 & 0.2753 & 0.5745 & 0.6342\end{array}$

$\begin{array}{lllllllllllllllllllll}\mathrm{C} 26 & 0.4137 & 0.4104 & 0.3064 & 0.5486 & 0.2065 & 0.3346 & 0.3579 & 0.2555 & 0.0000 & 0.4941 & 0.3741 & 0.2855 & 0.2671 & 0.1800 & 0.4404 & 0.4889\end{array}$

$\begin{array}{lllllllllllllllllll}\mathrm{C} 27 & 0.4003 & 0.3942 & 0.2339 & 0.5848 & 0.0738 & 0.2743 & 0.3206 & 0.1742 & 0.0000 & 0.5095 & 0.3346 & 0.2170 & 0.1738 & 0.1059 & 0.4356 & 0.5052\end{array}$

$\begin{array}{lllllllllllllllllll}\mathrm{C} 28 & 0.5890 & 0.6022 & 0.5174 & 0.7613 & 0.4061 & 0.5583 & 0.5420 & 0.4252 & 0.0000 & 0.6927 & 0.6052 & 0.4605 & 0.4705 & 0.2866 & 0.6442 & 0.7219\end{array}$

$\begin{array}{lllllllllllllllllll}\mathrm{C} 29 & 0.3953 & 0.3895 & 0.2535 & 0.5567 & 0.1219 & 0.2879 & 0.3255 & 0.1990 & 0.0000 & 0.4911 & 0.3383 & 0.2359 & 0.2032 & 0.1294 & 0.4254 & 0.4840\end{array}$ \begin{tabular}{lllllllllllllllll}
$\mathrm{C} 30$ & 0.5489 & 0.5583 & 0.4618 & 0.7208 & 0.3446 & 0.5019 & 0.4967 & 0.3765 & 0.0000 & 0.6521 & 0.5501 & 0.4126 & 0.4136 & 0.2517 & 0.5996 & 0.6743 \\
\hline
\end{tabular} 
Table 11. Cont.

\begin{tabular}{|c|c|c|c|c|c|c|c|c|c|c|c|c|c|c|c|c|}
\hline & $\mathrm{C} 17$ & C18 & C19 & $\mathrm{C} 20$ & $\mathrm{C} 21$ & $\mathrm{C} 22$ & $\mathrm{C23}$ & $\mathrm{C} 24$ & $\mathrm{C} 25$ & $\mathrm{C} 26$ & $\mathrm{C} 27$ & $\mathrm{C} 28$ & $\mathrm{C} 29$ & C30 & Eigenvectors & Ranking \\
\hline $\mathrm{C} 1$ & 0.5115 & 0.5255 & 0.4687 & 0.4983 & 0.5251 & 0.7418 & 0.5166 & 0.7187 & 0.4662 & 0.5863 & 0.5997 & 0.4110 & 0.6047 & 0.4511 & 0.1664 & 15 \\
\hline $\mathrm{C} 2$ & 0.5087 & 0.5236 & 0.4600 & 0.4931 & 0.5225 & 0.7594 & 0.5140 & 0.7331 & 0.4595 & 0.5896 & 0.6058 & 0.3978 & 0.6105 & 0.4417 & 0.1633 & 17 \\
\hline $\mathrm{C} 3$ & 0.5971 & 0.631 & 0.5782 & 0.61 & 0.6783 & 0.9122 & 0.614 & 0.8675 & 0.5412 & 0.6936 & 0.7661 & 0.4826 & 0.7465 & 0.5382 & 0.2006 & 8 \\
\hline $\mathrm{C} 4$ & 0.3732 & 0.3683 & 0.2759 & 0.3136 & 0.3159 & 0.6025 & 0.3648 & 0.5881 & 0.3219 & 0.4514 & 0.4152 & 0.2387 & 0.4433 & 0.2792 & 0.1031 & 28 \\
\hline $\mathrm{C} 5$ & 0.6910 & 0.1402 & 0.7205 & 0.7545 & 0.8540 & 1.0000 & 0.7219 & 0.9801 & 0.6349 & 0.7935 & 0.9262 & 0.5939 & 0.8781 & 0.6554 & 0.2454 & $J$ \\
\hline C6 & 0.5670 & 0.5963 & 0.5323 & 0.5725 & 0.6289 & 0.8855 & 0.5810 & 0.8422 & 0.5089 & 0.6654 & 0.7257 & 0.4417 & 0.7121 & 0.4981 & 0.1857 & 10 \\
\hline $\mathrm{C} 7$ & 0.5599 & 0.5827 & 0.5304 & 0.5618 & 0.6022 & 0.8198 & 0.5706 & 0.7887 & 0.5115 & 0.6421 & 0.6794 & 0.4580 & 0.6745 & 0.5033 & 0.1862 & 9 \\
\hline $\mathrm{C} 8$ & 0.6592 & 0.698 & 0.668 & 0.6970 & 0.7612 & 0.939 & 0.6811 & 0.8982 & 0.6119 & 0.7445 & 0.8258 & 0.574 & 0.8010 & 0.6235 & 0.2312 & 4 \\
\hline C9 & 1.0000 & 10000 & 1.0000 & 1.0000 & 10000 & 1.0000 & 10000 & 1.0000 & 1.0000 & 10000 & 10000 & 10000 & 10000 & 1.0000 & 0.3700 & 1 \\
\hline $\mathrm{C} 10$ & 0.428 & 0.431 & 0.353 & 0.388 & 0.4008 & 0.659 & 0.4258 & 0.6417 & 0.379 & 0.5059 & 0.4905 & 0.307 & 0.508 & 0.3479 & 0.1284 & 24 \\
\hline $\mathrm{C} 11$ & 0.5286 & 0.5506 & 0.4751 & 0.5172 & 0.5616 & 0.8389 & 0.5376 & 08001 & 0.4699 & 0.6259 & 0.6654 & 0.3948 & 0.6617 & 0.4499 & 0.1675 & 14 \\
\hline $\mathrm{C} 12$ & 0.629 & 0.664 & 0.6275 & 0.65 & 0.714 & 0.905 & 0.648 & 0.866 & 0.581 & 0.71 & 0.7830 & 0.53 & 0.764 & 0.5874 & 0.2177 & 6 \\
\hline $\mathrm{C} 13$ & 0.6354 & 0.6767 & 0.6353 & 0.6706 & 07455 & 0.9586 & 0.6578 & 0.9094 & 0.5802 & 0.7329 & 0.8262 & 05205 & 0.7968 & 0.5864 & 0.2188 & 5 \\
\hline C14 & 0.7572 & 0.7953 & 0.790 & 0.80 & 0.858 & 0.964 & 0.780 & 0.903 & 0.72 & 0.82 & 0.8941 & 0.71 & 0.870 & 0.7483 & 0.2762 & 2 \\
\hline $\mathrm{C} 15$ & 0.4768 & 0.4873 & 0.4137 & 0.4495 & 0.4732 & 0.7310 & 0.4789 & 0.7062 & 0.4255 & 0.5596 & 0.5644 & 0.3558 & 0.5746 & 0.4004 & 0.1481 & 22 \\
\hline C16 & 0.4225 & 0.4251 & 0.3289 & 0.3714 & 0.3843 & 0.6910 & 0.4186 & 0.6676 & 0.3658 & 0.5111 & 0.4948 & 0.2781 & 0.5160 & 0.3257 & 0.1205 & 27 \\
\hline C17 & 0.5000 & 05113 & 04581 & 04850 & 05071 & 0.7090 & 0.5037 & 0.6899 & 04582 & 05687 & 05752 & 04070 & 05823 & 0.4432 & 0.1630 & 18 \\
\hline C18 & 0.4887 & 0.5000 & 0.4357 & 0.4676 & 0.4914 & 0.1241 & 0.4917 & 0.7021 & 0.4413 & 0.5658 & 0.5723 & 0.3799 & 0.5001 & 0.4211 & 0.1555 & 20 \\
\hline C19 & 0.5419 & 0.5643 & 0.5000 & 0.5369 & 0.5795 & 0.8285 & 0.5517 & 0.7935 & 0.4879 & 0.6321 & 0.6703 & 0.4232 & 0.6662 & 0.4737 & 0.1759 & 13 \\
\hline C20 & 0.5150 & $0-5221$ & $0-1621$ & C 5000 & 0.5345 & ? 7000 & C 5215 & 0 7501 & 04618 & 0.6028 & 0.0200 & 0.3940 & 06780 & 0.4424 & 0.1641 & 16 \\
\hline $\mathrm{C} 21$ & 0.4929 & 0.5086 & 0.4205 & 0.4655 & 0.5000 & 0.8018 & 0.4974 & 0.7660 & 0.4324 & 0.5910 & 0.6131 & 0.3482 & 0.6178 & 0.4031 & 0.1498 & 21 \\
\hline C22 & 0.2910 & 02752 & 01715 & 0 & 1087 & 05000 & (2750 & 04032 & (2) & 03651 & 03010 & 01488 & 02100 & 0.1859 & 0.0693 & 30 \\
\hline $\mathrm{C} 23$ & 0.4963 & 0.5083 & 0.4483 & 0.4785 & 0.5026 & 0.7248 & 0.5000 & 0.7029 & 0.4507 & 0.5710 & 0.5790 & 0.393 & 0.5864 & 0.4329 & 0.1596 & 19 \\
\hline $\mathrm{C} 24$ & 2101 & 02070 & 02065 & (2400 & 02340 & 05067 & (207 20 & 05000 & 02630 & ( 2706 & 02256 & 01816 & 0.5390 & 0.2163 & 0.0799 & 29 \\
\hline $\mathrm{C} 25$ & 0.5418 & 0.5587 & 0.5121 & 0.5382 & 0.5676 & 0.7598 & 0.5493 & 0.7370 & 0.5000 & 0.6122 & 0.6329 & 0.4537 & 0.6339 & 0.4914 & 0.1808 & 12 \\
\hline C26 & 04313 & 04241 & 03670 & 03072 & 04000 & 06340 & 04200 & 06204 & 03878 & 05000 & 0.4850 & 0.3262 & 05016 & 0.3617 & 0.1332 & 23 \\
\hline $\mathrm{C} 27$ & 0.424 & 0.4277 & 0.3297 & 0.373 & 0.3869 & 0.6990 & 0.4210 & 0.6744 & 0.367 & 0.515 & 0.5000 & 0.277 & 0.520 & 0.3263 & 0.1208 & 26 \\
\hline C28 & 0.5930 & 06201 & 0.5768 & 0.6060 & 0.6518 & 0.8512 & 0.6069 & 0.8184 & 0.5463 & 0.6738 & 0.7224 & 0.5000 & 07122 & 0.5449 & 0.2015 & 7 \\
\hline C29 & 0.4177 & 0.4193 & 0.3338 & 0.371 & 0.3822 & 0.6591 & 0.4136 & 0.6402 & 0.3661 & 0.4984 & 0.4791 & 0.2878 & 0.5000 & 0.3304 & 0.1220 & 25 \\
\hline C30 & 0.5568 & 0.5789 & 0.5263 & 0.5576 & 0.5969 & 0.8141 & 0.5671 & 0.7837 & 0.5086 & 0.6383 & 0.6737 & 0.4551 & 0.6696 & 0.5000 & 0.1850 & 11 \\
\hline
\end{tabular}

\section{Theoretical and Managerial Implications}

This section presents the theoretical contributions that relate to innovation and SBMCC and provides managerial implications for practice.

\subsection{Theoretical Implications}

Innovation was found to contribute to SBMCC, as in previous studies [15,20,79]. This study contributes to the literature by prioritizing the capabilities and competencies, thereby revealing a better understanding of innovation in these areas and of SBMCC. This study provides evidence that suggests that innovation in technology capabilities, networking and social capabilities, and the capabilities for learning and developing should be the priorities for a sustainable business. 
Firms that have the potential to make products using technologies that challenge the current technology have technology capabilities [80]. Technology capabilities contribute to firm innovation in a highly competitive environment by helping to satisfy customer demand for product and service innovation [81]. Moreover, it affects the business model: Business sustainability drives the firm to adopt management innovation [21,82]. Management innovation refers to the generation or adoption of management processes, practices, structures or techniques that are new to the firm and that affect its performance in terms of innovation, productivity and competitiveness [83,84]. Hence, it changes the way management, techniques and procedures are used to accomplish a specific task or goal and improves on traditional processes and practices. These changes can create sustainable competitive advantages that lead to economic success [85-87].

Previous studies have widely noted that to proceed to a stage of sustainable business, it is necessary to involve a range of actors, including government, societal, nongovernmental organizations, and the community [23,88-91]. Different actors in society and in networks provide learning opportunities, innovation, positive influences, business opportunities and even solutions for complex problems [63]. Thus, innovation of SBMCC requires networking capabilities for collaboration, which facilitates changes at all levels — both inside and outside of the firm [92]. In terms of technology activities, strong networking and social capabilities benefit technology innovation because of the collaboration among actors in a network, as innovation can be achieved by implementing additional capabilities from outside sources [93]. In particular, collaborating with universities and research institutions positively affects product innovation [94]. Specifically, such collaboration allows firms to acquire new scientific knowledge from universities and research institutions, which might benefit product or process innovation. By contrast, failing to understand the benefits of this collaboration may lead to a firm lagging behind in terms of competitive advantage [95].

Learning is the basis of the enhancement of sustainable competitive advantage [96]. Firms that have learning and development capabilities stand a better chance of sensing and efficiently adapting to trends in the marketplace [97]. Capabilities for learning and developing facilitate innovation, which in turn significantly influences the achievement of sustainable business [22]. It ensures business sustainability, i.e., that successes and best practices are transferred and translated into action. In addition, creativity, innovation and adaptability can be leveraged. Learning can be approached from different levels, such as the individual, group, organization and network levels [98,99]. Networks such as industrial associations and industrial forums as discussed above or even competitors can promote innovation for sustainable business. The advantages of collaborating and learning from competitors are sharing knowledge and skills, solving common problems, and gaining more knowledge about competitor strategies [100,101].

\subsection{Managerial Implications}

This section presents several practical implications. The ranking of the most important criteria are provided in the results section, which offers several managerial insights for the hotel industry. Given the highly competitive nature of the hotel industry, a basic understanding of innovation is necessary for SBMCC. Next, we discuss the implications derived from this study.

The top-ranking criterion is to develop social relations with (culturally) unfamiliar actors inside or outside the organization for information gathering, experimentation and negotiation (C9). Sustainable business may require a re-thinking of the terms of competition and collaboration among the actors 
engaged in entire networks. The hotel must facilitate cooperation among the actors in a network, creating a basis for new product and service development and innovation as well as developing advantages associated with lower costs, the preemption of competitors, and a favorable future market position, which can help the hotel achieve increased legitimacy through transparency and collaboration with stakeholders [21,64]. Therefore, management should encourage and facilitate social relations for the sake of improving their products and services; for example, building relationships with research institutions for learning and innovation; building relationships with governments and industrial associations that assist firms in predicting and adapting to new policies and trends in business; collaborating with travel agencies for stable customer sources; and even collaborating with technology-based app developers to receive support from customer app searches.

Upgrading and integrating technology capabilities may benefit new product development and marketing (C14) in several ways; for example, it may decrease the cost of products and services while improving the efficient use of resources [102,103]. Some noteworthy advanced technologies include social media, mobile-friendly content, new technology for traditional points of sale, etc., and these new technologies can help a hotel advertise its products and services to potential customers regardless of distance with low-cost systems for searching and purchasing products. Moreover, technology may assist in changing a firm's business model [104]. Technology is the basis of e-commerce, which is now considered the future of the hotel industry. Cloud computing reduces costs, strengthens security, and allows easy access to information. Currently, mobile phones and tablets can be used to book rooms, plan trips, and search for hotels. Thus, both the accessibility and security of information are critical in business. Hotels that aim to achieve sustainability should consider continuous upgrades in, integration with, and exploitation of new technology.

The ability to think inventively (C5) is an individual skill that is crucial for innovation and that is directed toward finding new solutions to problems. This capability should be strongly developed, as the ability to think innovatively assumes the role of idea generator to achieve change and innovation in hotels. Management and employees with a competency for inventive thinking form the basis of innovation. Therefore, hotels seek and hire inventive employees to improve innovation. Moreover, support and encouragement from management can assist individual employees with thinking inventively, thus creating benefits for firms in terms of innovation and problem-solving. In practice, training courses in problem-solving techniques, such as brainstorming, have proven effective in leveraging inventive thinking and creativity on the part of employees. As employees are the main actors in processing and delivering hotel service to customers, an inventive employee can derive solutions to complex problems and use work experience as a basis for innovation.

Building trust and a shared vision and agreement on basic values (C8) is necessary for collaboration; however, it involves a highly diverse group of stakeholders, which is problematic because of opposing interests and perspectives [21]. Collaboration within a network requires a shared vision and values to achieve uniformity in action. In terms of supporting employees, management is encouraged to be open and responsive to different perspectives - as opposed to closed management attitudes - because it might lead to new knowledge bases for sustainable business management and thus benefit the common agreement on basic values. To achieve openness, managers must be willing to relinquish some control, to find a balance between managing and being managed, and to empower employees. Employees with 
more freedom are also free to create innovative solutions that can make the firm more competitive. Hence, the advantages of empowerment in the firm should be emphasized.

Finally, new approaches to knowledge regarding management functions and new processes that produce changes in organizational strategies, structures, administrative procedures, and systems (C13) foster firm process innovation and management innovation. In the hotel industry, process innovation focuses on eliminating waste in delivering services to customers. In this case, implementing technology advancements can improve service quality by providing greater convenience and better experiences to customers while minimizing managerial costs. Currently, the most remarkable technologies that have improved hotel service in terms of process and management are virtual booking and cloud computing. These technologies might help hotels cut costs by reducing the upfront costs associated with setting up the hotel agent, hardware, and operations. More specifically, systems that can be migrated to the cloud and accessed on electronic equipment are desirable to increase customer convenience while improving managerial efficiency and effectiveness. Moreover, the minor details that should be considered include Internet access, sufficient electronics support (adequate and easy-to-reach electrical outlets), etc.

\section{Conclusions}

This study employs IVTFNs and the GRA method to understand innovations in SBMCC by identifying and ranking capabilities and competencies. The capabilities and competencies regarding sustainable business management are presented as several aspects, including competencies for systemic thinking; networking and social capabilities; technology capabilities; capabilities for learning and developing; and capabilities for integrating business, environmental problems, social problems, perspectives and information. These five aspects were considered and evaluated in enhancing the capabilities and competencies for firms to achieve sustainability. The degrees of importance of the aforementioned aspects and criteria are determined by linguistic preference number and the ranking of eigenvectors.

The findings confirm that innovation was found to support SBMCC; this finding implies that innovation in the aspects of technology capabilities, networking and social capabilities, and capabilities for learning and development should be more highly prioritized than other aspects in management decision-making. Specifically, technology capabilities are particularly important in the innovation of management, process, and procedure, all of which can lead to economic success and sustainable business. In the hotel industry, as a result of globalization, the future of doing business is providing service to customers regardless of geographic barriers. Technology enables this possibility for hotels because of e-commerce, which provides virtual reservations, check-in, and ordering. This affirms the sustainability of the business even within the highly competitive environment of the hotel industry. Furthermore, to achieve a sustainable business, a variety of actors must be involved, including government, civic society, nongovernmental organizations, and the community; hence, strong networking and social capabilities require more attention from management. This benefits sustainable business management by fostering long-term collaboration and agreement among the actors and stakeholders in a firm network, which can lead to synergies for development. To exploit a firm's network and social capabilities, the capabilities for learning and development must be translated into action; in addition, innovation is necessary for competitiveness and business success.

This study has contributed to understanding innovation in SBMCC. In practice, establishing a variety of social relations is necessary for a hotel to better adapt to the changes in the business environment. 
In addition, applying technology upgrades and integration improves customers' convenience and also plays an important role in business. Technology not only changes management functions and processes, but also reduces the costs, improves service speed, and enhances customer satisfaction. However, the idea of improvements may originate from an individual employee or at the management level; thus, the competency to think inventively is required to encourage and spread the changes to all levels of process and management. Moreover, building trust, a shared vision, and agreement on basic values drives the actors in a network that is dedicated to the common development of sustainable business. In particular, managers are advised to stimulate the entrepreneurial spirit and thinking among employees [105].

This study has certain limitations. First, it was conducted using the extant innovation and SBMCC literature to identify the relevant aspects and criteria; thus, the set of aspects and criteria may not be comprehensive. Second, the sample collection focused only on Taiwanese hotels; hence, external generalizability is limited. Future research would benefit from multi-industry data, which might address potential problems with generalizability. Similarly, the expert sample might be categorized into different industries that may benefit from the comparison and examination of the effective aspects and criteria. Furthermore, to promote and deepen the understanding of SBMCC, additional studies must be investigated to uncover additional valuable aspects and criteria to refine the accuracy of the analysis.

\section{Acknowledgments}

The authors of this study would like to thank Hong Thuy Tien Nguyen, Kim Truong Pham, Phuong Quy Huynh and Quoc Cuong Dang for their efforts. Their contributions provided a significant basis for the formulation of this study.

\section{Author Contributions}

Kuo-Jui Wu and Ching-Jong Liao found the phenomenon in the industry and conducted this study. Ming-Lang Tseng and Pei-Jay Chou proposed the research method and finalized the analysis to overcome the uncertainty and incomplete information. Then Kuo-Jui Wu combined the research design and analysis to write the paper for enhancing the understanding of SBMCC. Consequently, all authors read and approved the final manuscript.

\section{Conflicts of Interest}

The authors declare no conflict of interest.

\section{References}

1. Tseng, C.; Kuo, H.; Chou, S. Configuration of innovation and performance in the service industry: Evidence from the Taiwanese hotel industry. Serv. Ind. J. 2008, 28, 1015-1028.

2. Gu, H.; Ryan, C.; Yu, L. The changing structure of the Chinese hotel industry: 1980-2012. Tour. Manag. Perspect. 2012, 4, 56-63.

3. Grissemann, U.S.; Pikkemaat, B.; Weger, C. Antecedents of innovation activities in tourism: An empirical investigation of the Alpine hospitality industry. Tourism 2013, 61, 7-27. 
4. Zhou, K.Z.; Brown, J.R.; Dev, C.S.; Agarwal, S. The effects of customer and competitor orientations on performance in global markets: A contingency analysis. J. Int. Bus. Stud. 2007, 38, 303-319.

5. Chen, J.S.; Tsou, H.T.; Huang, Y.H. Service delivery innovation: Antecedents and impact on firm performance. J. Serv. Res. 2009, 12, 36-55.

6. Den Hertog, P. Knowledge-intensive business services as co-producers of innovation. Int. J. Innov. Manag. 2000, 4, 491-528.

7. Gebauer, H.; Gustafsson, A.; Witell, L. Competitive advantage though service differentiation by manufacturing companies. J. Bus. Res. 2011, 64, 1270-1280.

8. Damanpour, F. An integration of research findings of effects of firm size and market competition on product and process innovations. Br. J. Manag. 2010, 21, 996-1010.

9. Hjalager, A.M. A review of innovation research in tourism. Tour. Manag. 2010, 31, 1-12.

10. Nybakk, E. Learning orientation, innovativeness and financial performance in traditional manufacturing firms: A higher-order structural equation model. Int. J. Innov. Manag. 2012, 16, 1-33.

11. Rhee, J.; Park, T.; Lee, D.H. Drivers of innovativeness and performance for innovative SMEs in South Korea: Mediation of learning orientation. Technovation 2010, 30, 65-75.

12. Delgado-Verde, M.; Martín-de Castro, G.; Navas-López, J.E. Organizational Knowledge Assets and Innovation Capability: Evidence from Spanish Manufacturing Firms. J. Intell. Cap. 2011, 12, 5-19.

13. Harper, S.M.; Becker, S.W. On the leading edge of innovation: A comparative study of innovation practices. South. Bus. Rev. 2004, 29, 1-15.

14. Walker, R.M.; Damanpour, F.; Devece, C.A. Management innovation and organizational performance: The mediating effect of performance management. J. Public Adm. Res. Theory 2011, 21, 367-386.

15. McEvily, B.; Zaheer, A. Bridging ties: A source of firm heterogeneity in competitive capabilities. Strateg. Manag. J. 2003, 20, 1133-1156.

16. Teece, D.; Pisano, G.; Shuen, A. Dynamic capabilities and strategic management. Strateg. Manag. J. 1997, 18, 509-533.

17. Zahra, S.A. Entrepreneurial risk taking in family firms. Fam. Bus. Rev. 2005, 18, 23-40.

18. Colquitt, J.A.; Scott, B.A.; LePine, J.A. Trust, trustworthiness, and trust propensity: A meta-analytic test of their unique relationships with risk-taking and job performance. J. Appl. Psychol. 2007, 92, 909-927.

19. Delgado, M.; Porter, M.E.; Stern, S. Clusters and entrepreneurship. J. Econ. Geogr. 2010, 10, 495-518.

20. Orfila-Sintes, F.; Mattsson, J. Innovation behavior in the hotel industry. Omega 2009, 37, 380-394.

21. Van Kleef, J.A.G.; Room, N.J. Developing capabilities and competencies for sustainable business management as innovation: A research agenda. J. Clean. Prod. 2007, 15, 38-41.

22. Weerawardena, J.; Mavondo, F.T. Capabilities, innovation and competitive advantage. Ind. Mark. Manag. 2011, 40, 1220-1223.

23. Bodin, Ö.; Crona, B.I. The role of social networks in natural resource governance: What relationship patterns make a difference? Glob. Environ. Chang. 2009, 19, 366-374. 
24. Tseng, M.L.; Wang, R.; Chiu, A.S.F.; Geng, Y.; Lin, Y.H. Improving performance of green innovation practices in uncertainty. J. Clean. Prod. 2013, 40, 71-82.

25. Wong, Y.P.; Tseng, M.L.; Tan, K. A business process management capabilities perspective on organization performance. Total Qual. Manag. Bus. Excell. 2014, 25, 602-617.

26. Kuo, Y.; Yang, T.; Huang, G.W. The use of grey relational analysis in solving multiple attribute decision-making problems. Comput. Ind. Eng. 2008, 55, 80-93.

27. Tseng, M.L. Using linguistic preferences and grey relational analysis to evaluate the environmental knowledge management capacity. Expert Syst. Appl. 2010, 37, 70-81.

28. Onsel, S.; Ulengin, F.; Kaba, O. A cluster-based approach for the innovation assessment of countries. In Proceedings of the Engineering Management Conference, Estoril, Portugal, 28-30 June 2008; pp. 1-5.

29. McFadzean, E.; O’Loughlin, A.; Shaw, E. Corporate entrepreneurship and innovation part 1: The missing link. Eur. J. Innov. 2005, 8, 350-372.

30. Oke, A. Innovation types and innovation management practices in service companies. Int. J. Oper. Prod. Manag. 2007, 7, 564-587.

31. Edquist, C. Systems of innovation approaches - Their emergence and characteristics. In Systems of Innovation-Technologies, Institutions and Organizations; Pinter Publishers/Cassell Academic: London, UK, 1997; pp. 1-35.

32. Johannesen, J.; Olsen, B. Systemic knowledge processes, innovation and sustainable competitive advantages. Kybernetes 2009, 38, 559-580.

33. Liao, T.; Price, J. Innovation investments, market engagement and financial performance: A study among Australian manufacturing SMEs. Res. Policy 2010, 39, 117-125.

34. Van de Ven, A.; Engleman, R. Central problems in managing corporate innovation and entrepreneurship. In Advances in Entrepreneurship, Firm Emergence and Growth 7; Emerald Group Publishing: West Yorkshire, UK, 2007; pp. 47-72.

35. Hallstedt, S.I.; Thompson, A.W.; Lindahl, P. Key elements for implementing a strategic sustainability perspective in the product innovation process. J. Clean. Prod. 2013, 51, 277-288.

36. Hansen, E.G.; Grosse-Dunker, F.; Reichwald, R. Sustainability innovation cube-A framework to evaluate sustainability-oriented innovations. Int. J. Innov. Manag. 2009, 13, 683-713.

37. Schaltegger, S.; Wagner, M. Sustainable entrepreneurship and sustainability innovation: Categories and interactions. Bus. Strategy Environ. 2011, 20, 222-237.

38. Dutta, S.; Narasimhan, O.; Rajiv, S. Conceptualizing and measuring capabilities: Methodology and empirical application. Strateg. Manag. J. 2005, 26, 277-285.

39. Campion, M.A.; Fink, A.A.; Ruggeberg, B.J.; Carr, L.; Phillips, G.M.; Odman, R.B. Doing Competencies Well: Best Practices in Competency Modelling. Pers. Psychol. 2011, 64, 225-262.

40. Leblanc, R.; James, G. Inside the Boardroom: How Boards Really Work and the Coming Revolution in Corporate Governance; John Wiley \& Sons: Hoboken, NJ, USA, 2010.

41. Markus, L.H.; Cooper-Thomas, H.D.; Allpress, K.N. Confounded by Competencies? An Evaluation of the Evolution and Use of Competency Models. N. Z. J. Psychol. 2005, 34, 117-126.

42. Yusoff, W.F.W.; Armstrong, A. What Competencies Should Directors Possess? Malaysia Perspective. Int. J. Bus. Manag. 2012, 7, 142-149. 
43. Lim, J.H.; Stratopoulos, T.C.; Wirjanto, T.S. Role of IT executives in the firm's ability to achieve competitive advantage through IT capability. Int. J. Account. Inf. Syst. 2012, 13, 21-40.

44. Nobre, F.S.; Walker, D.; Harris, R.J. Technological, Managerial and Organizational Core Competencies: Dynamic Innovation and Sustainable Development. IGI Global: Hershey, PA, USA, 2012.

45. Matos, S.; Silvestre, B. Managing stakeholder relations when developing sustainable business models: The case of the Brazilian energy sector. J. Clean. Prod. 2013, 45, 61-73.

46. Schoenherr, T. The role of environmental management in sustainable business development: A multi-country investigation. Int. J. Prod. Econ. 2012, 140, 116-128.

47. Wu, K.J.; Liao, C.J.; Tseng, M.L.; Chiu, A.S.F. Exploring decisive factors in green supply practices under uncertainty. Int. J. Prod. Econ. 2015, 159, 147-157.

48. Deng, J.L. Introduction to grey system theory. J. Grey Syst. 1989, 1, 1-24.

49. Lin, Y.H.; Lee, P.C.; Chang, T.P. Practical expert diagnosis model based on the grey relational analysis technique. Expert Syst. Appl. 2009, 36, 1523-1528.

50. Wei, G.U. GRA method for multiple attribute decision making with incomplete weight information in intuitionistic fuzzy setting. Knowl. Based Syst. 2010, 23, 243-247.

51. Kapsali, M. Systems thinking in innovation project management: A match that works. Int. J. Proj. Manag. 2011, 29, 396-407.

52. Lansdown, T.C.; Stephens, A.N.; Walker, G.H. Multiple driver distractions: A systemic transport problem. Accid. Anal. Prev. 2015, 74, 360-367.

53. Joham, C.; Metcalfe, M.; Sastrowardoyo, S. Project conceptualization using pragmatic methods. Int. J. Proj. Manag. 2009, 27, 787-794.

54. Pourdehnad, G. Synthetic (integrative) project management, an idea whose time has come. Bus. Strategy Ser. 2007, 8, 426-434.

55. Salmon, P.M.; McClure, R.; Stanton, N.A. Road transport in drift? Applying contemporary systems thinking to road safety. Saf. Sci. 2012, 50, 1829-1838.

56. Kline, N. More Time to Think: The Power of Independent Thinking; Octopus: London, UK, 2015.

57. Brodin, E.M. The Critical and Creative Thinking Nexus: Learning Experiences of Doctoral Students. In Studies in Higher Education; Routledge: London, UK, 2014.

58. Levine, M. A Mind at a Time: How Every Child Can Succeed? Simon \& Schuster: New York, NY, USA, 2012.

59. Colvin, W.; Lyden, S.; Barra, B.A.L. Attracting Girls to Civil Engineering through Hands-on Activities that Reveal the Communal Goals and Values of the Profession. J. Leadersh. Manag. Eng. 2013, 13, 35-41.

60. Chang, Y.C.; Chang, H.T.; Chi, H.R.; Chen, M.H.; Deng, L.L. How do established firms improve radical innovation performance? The organizational capabilities view. Technovation 2012, 32, $441-451$.

61. Erzurumlu, S.S.; Erzurumlu, Y.O. Sustainable mining development with community using design thinking and multi-criteria decision analysis. Resour. Policy 2014, doi:10.1016/j.resourpol.2014.10.001. 
62. Petrini, M.; Pozzebon, M. Managing sustainability with the support of business intelligence: Integrating socio-environmental indicators and organisational context. J. Strateg. Inf. Syst. 2009, 18, 178-191.

63. Christiansen, A.; Prescott, T.; Ball, J. Learning in action: Developing safety improvement capabilities through action learning. Nurse Educ. Today 2014, 34, 243-247.

64. Yang, Y.; Narayanan, V.K.; Zahra, S. Developing the selection and valuation capabilities through learning: The case of corporate venture capital. J. Bus. Ventur. 2009, 24, 261-273.

65. O’Connor, K.M.; Gladstone, E. How social exclusion distorts social network perceptions? Soc. Netw. 2015, 40, 123-128.

66. Hsieh, M.H.; Tsai, K.H. Technological capability, social capital and the launch strategy for innovative products. Ind. Mark. Manag. 2007, 36, 493-502.

67. Galende, J.; de la Fuente, J.M. Internal factors determining a firm's innovative behavior. Res. Policy 2003, 32, 715-736.

68. Kafetzopoulos, D.; Psomas, E. The impact of innovation capability on the performance of manufacturing companies: The Greek case. J. Manuf. Technol. Manag. 2015, 26, 104-130.

69. Finin, T.; Ding, L.; Zou, L. Social networking on the semantic web. Learn. Organ. 2005, 12, 418-435.

70. McGrath, H.; O'Toole, T. A cross-cultural comparison of the network capability development of entrepreneurial firms. Ind. Mark. Manag. 2014, 43, 897-910.

71. Dyllick, T.; Hockerts, K. Beyond the business case for corporate sustainability. Bus. Strategy Environ. 2002, 11, 130-141.

72. Young, W.; Tilley, F. Can businesses move beyond efficiency? The shift toward effectiveness and equity in the corporate sustainability debate. Bus. Strategy Environ. 2006, 15, 402-415.

73. European Commission. Sustainable production, challenges and objectives for EU research policy. In Report of the Expert Group on Competitive and Sustainable Production \& Related Service Industries in Europe in the Period to 2020; EUR19880; European Commission: Brussel, The Netherland, 2001.

74. Letki, N.; Mierina, I. Getting support in polarized societies: Income, social networks, and socioeconomic context. Soc. Sci. Res. 2015, 49, 217-233.

75. Deng, J.L. Control problems of grey systems. Syst. Control Lett. 1982, 1, 288-294.

76. Zhang, S.F.; Liu, S.Y.; Zhai, R.H. An extended GRA method for MCDM with interval-valued triangular fuzzy assessments and unknown weights. Comput. Ind. Eng. 2011, 61, 1336-1341.

77. Li, D.-F.; Wang, Y.-C.; Liu, S.; Shan, F. Fractional programming methodology for multi-attribute group decision-making using IFS. Appl. Soft Comput. 2009, 9, 219-225.

78. World Tourism Organization. UNWTO Tourism Highlights, 2014. Available online: http://mkt.unwto.org/publication/unwto-tourism-highlights-2014-edition (accessed on 15 February 2015).

79. Grissemann, U.S.; Plank, A.; Brunner-Sperdin, A. Enhancing business performance of hotels: The role of innovation and customer orientation. Int. J. Hosp. Manag. 2013, 33, 347-356.

80. Veryzer, R.W. Discontinuous innovation and the new product development process. J. Prod. Innov. Manag. 1998, 15, 304-321.

81. Pantano, E.; Viassone, M. Consumers' expectation of innovation: Shift retail strategies for more attractive points of sale. Int. J. Digit. Content Technol. Appl. 2012, 16, 455-461. 
82. Hecker, A.; Ganter, A. The influence of product market competition on technological and management innovation: Firm-level evidence from a large scale survey. Eur. Manag. Rev. 2013, $10,17-33$.

83. Birkinshaw, J.; Hamel, G.; Mol, M.J. Management innovation. Acad. Manag. Rev. 2008, 33, 825-845.

84. Volberda, H.W.; van den Bosch, F.A.J.; Heij, C.V. Management innovation: Management as fertile ground for innovation. Eur. Manag. Rev. 2013, 10, 1-15.

85. D'Amato, A.; Roome, N. Leadership of organizational change toward an integrated model of leadership for corporate responsibility and sustainable development: A process model of corporate responsibility beyond management innovation. Corp. Govern. 2009, 9, 421-434.

86. Hamel, G. The why, what and how of management innovation. Harv. Bus. Rev. 2006, 84, 72-84.

87. Wu, L.Y. Which companies should implement management innovation? A commentary essay. J. Bus. Res. 2010, 63, 321-323.

88. Meadows, J.; Herbohn, J.; Emtage, N. Engaging Australian small-scale lifestyle landowners in natural resource management programmes-Perceptions, past experiences and policy implications. Land Use Policy 2014, 36, 618-627.

89. Phillips, P.S.; Tudor, T.L.; Bird, H.; Bates, M.B. A critical review of a key Waste Strategy initiative in England: Zero Waste Places Projects 2008-2009. Resour. Conserv. Recycl. 2011, 55, 335-343.

90. Stark1, M.; Brunner, N.; López, E.; Martínez-Ruiz, J.L. A planning-oriented sustainability assessment framework for peri-urban water management in developing countries. Water Resour. 2013, 47, 7175-7183.

91. Weber, T.C.; Allen, W.L. Beyond on-site mitigation: An integrated multiscaleapproach to environmental mitigation and stewardship for transportation projects. Lands. Urban Plan. 2010, 96, 240-256.

92. Clarke, S.; Roome, N.J. Sustainable business: Learning-action networks as organizational assets. Bus. Strategy Environ. 1999, 8, 296-310.

93. Becker, W.; Dietz, J. R\&D cooperation and innovation activities of firms-Evidence for the German manufacturing industry. Res. Policy 2004, 33, 209-223.

94. Nieto, M.J.; Santamaría, I. The importance of diverse collaboration networks for the novelty of product innovation. Technovation 2007, 27, 367-377.

95. Spencer, J. Firms' knowledge-sharing strategies in the global innovation system: Empirical evidence from the flat panel display industry. Strateg. Manag. J. 2003, 24, 217-233.

96. Brockmand, B.; Morgan, F. The role of existing knowledge in new product innovativeness and performance. Decis. Sci. 2003, 32, 385-419.

97. Tippins, M.J.; Sohi, R.S. IT competency and firm performance: Is organizational learning a missing link? Strateg. Manag. J. 2003, 24, 745-761.

98. Bontis, N.; Crossan, M.; Hulland, J. Managing organizational learning systems by aligning stocks and flows. J. Manag. Stud. 2002, 39, 437-469.

99. Knight, L. Network learning: Exploring learning by interorganizational networks. Hum. Relat. 2002, 55, 427-454.

100. Linn, T.A. Learning from the competition. J. Account. 1994, 177, 43-46.

101. Tether, B. Who co-operate for innovation, and why: An empirical analysis. Res. Policy 2002, 31, 947-967. 
102. Bodhani, A. Shops offer the e-tail experience. Eng. Technol. 2012, 7, 46-49.

103. Tajeddini, K.; Trueman, M. Perceptions of innovativeness among Iranian hotel managers. J. Hosp. Tour. Technol. 2014, 5, 62-77.

104. Boons, F.A.A.; Lüdeke-Freund, F. Business models for sustainable innovation: State-of-the-art and steps towards a research agenda. J. Clean. Prod. 2013, 45, 9-19.

105. Tajeddini, K. Exploring the antecedents of effectiveness and efficiency. Int. J. Hosp. Manag. 2015, $49,125-135$.

(C) 2015 by the authors; licensee MDPI, Basel, Switzerland. This article is an open access article distributed under the terms and conditions of the Creative Commons Attribution license (http://creativecommons.org/licenses/by/4.0/). 